Journal of Experimental Psychology:

Human Perception and Performance

1999, vol. 25, No. 1, 83-101.

\title{
Attention accesses multiple reference frames: Evidence from visual neglect
}

\author{
Marlene Behrmann \\ Carnegie Mellon University
}

\author{
Steven P. Tipper \\ University of Wales
}

\begin{abstract}
Research with normal subjects has demonstrated that mechanisms of selective attention can simultaneously gain access to internal representations of spatial information defined with respect to both location- and object-based frames of reference. We demonstrate that patients with unilateral spatial neglect following a right hemisphere lesion are poorer at detecting information on the contralateral left side in both location- and object-based spatial coordinates simultaneously. Moreover, the extent of the neglect is modulated by the probability of a target appearing in either reference frame; as the probability of sampling a target in a particular frame of reference increases, so does the severity of neglect in that frame. These findings suggest that attention can be flexibly and strategically assigned to a reference frame depending on the contingencies of the task.
\end{abstract}

This research was supported by a NIH FIRST award (MH 54246-01) to Marlene Behrmann and by a grant from the Higher Education Funding Council of Wales to Steve Tipper.

We thank John McGoldrick and James Nelson for help with various aspects of this research. We also thank Dr Bob Stowe of the VA Hospital, Highland Drive and Dr Graham Ratcliff of Harmarville Rehabilitation Center for referring the patients to us and the University of Pittsburgh Medical Center for providing access to patients. We are grateful to Dr N. Weikers for his interpretation of the CT scan results. We are grateful to Tom Carr, Glyn Humphreys, Rich Ivry, Bob Rafal and two anonymous reviewers for their constructive comments and suggestions.

Correspondence regarding this article should be addressed to either author:

Marlene Behrmann, Department of Psychology, Carnegie Mellon University, Pittsburgh, PA 15213-3890. email: behrmann+@ cmu .edu; phone (412) 268-2790; fax (412) 2682798 
Steven P. Tipper, Department of Psychology, University of Wales, Bangor, Gwynedd, Wales, UK, LL57 2DG. email: s.tipper@bangor.ac.uk; phone: (01248) 382095; fax (01248) 382599

The medium upon which selective attention mechanisms function has been vigorously debated. A dominant view has been that this medium is a spatial map of the environment which is internally represented. Attention is viewed as a spotlight (Broadbent, 1982; Posner, 1980) or zoom lens (Eriksen \& Schultz, 1979) that moves over this spatial map, facilitating the processing of stimuli within its beam (for recent review, see Egeth \& Yantis, 1997). An alternative, more recent account is that an objectbased frame of reference may be accessed by selective attention systems (Duncan, 1984; Kahneman \& Treisman, 1984) and considerable empirical evidence now exists favoring this perspective (for example, Baylis \& Driver, 1993; Behrmann, Zemel, \& Mozer, 1998; Kramer \& Jacobson, 1991; Kramer \& Watson, 1996; Kramer, Weber, \& Watson, 1997; Lavie \& Driver, 1996; Moore, Yantis, \& Vaughan, 1998; Reuter-Lorenz, Drain, \& Hardy-Morais, 1996; Weber, Kramer, $\&$ Miller, 1997). The motivation behind this alternative proposal arises from considerations about what functions attention has evolved to serve. Thus, attention appears to be a mechanism that selects a salient item from the essentially parallel visual perceptual system in the service of the serial motor system. Through the operation of such a mechanism, action may be directed towards one of the many objects that potentially evoke a response (see Tipper \& Weaver, 1996). On this account, selective attention is crucial for coherent goal-directed behavior which is directed towards particular objects at the appropriate times (Tipper, Weaver, \& Houghton, 1994).

The evidence that attention is not simply determined by the spatial relationships between stimuli, as a purely spatial model would predict, has come from a number of studies. For example, Eriksen and Eriksen (1974) showed that ignored distractor letters impaired responses to targets at fixation only when they were within 1 degree of the target. Such a result supports the notion that the zoom lens of attention has a limited resolution on the spatial map. However, several studies have shown that when objects were grouped together by common motion, interference could be produced even when the distractors were spatially distant from the target (Baylis \& Driver, 1992; Driver \& Baylis, 1989). Clearly such a result cannot be explained within a framework where a limited resolution attention mechanism simply accesses a spatial map; on the contrary, "higher" level object grouping is determining the performance of the selective attention system (see also Baylis \& Driver, 1993; Kramer \& Jacobson, 1991; Stuart, Maruff, \& Currie, 1997).

Initially it was suggested that object-based frames might suffice as the medium of attention (Tipper, Driver, \& Weaver, 1991). However, consideration of perceptual processes make this claim unlikely. As J.J. Gibson (1979) made clear, objects are not perceived in free-floating isolation, but rather the perception of object motion (Duncker, 1929; Wallach, 1959), 
location (Matin, Picoult, Stevens, Edwards, \& MacArthur, 1982), and depth (see Goldstein, 1996, for review) is determined by a background environmental context or frame. Subsequent work has indeed demonstrated that mechanisms of attention, such as inhibition, can act on both spatial- (or environmental context) and object-based frames of reference simultaneously. For example, when an object is cued, processing of subsequent information can be inhibited, a phenomenon referred to as inhibition of return (Abrams \& Dobkin, 1994; Posner \& Cohen, 1984). If an object that is initially cued then moves to a new location, the inhibition moves with the object, supporting the notion of objectbased inhibition mechanisms (Tipper et al., 1991). Importantly, however, processing of information at the location initially cued is also impaired independently of subsequent object motion, supporting the idea of locationbased inhibition mechanisms (Egly, Driver, \& Rafal, 1994; B. S. Gibson \& Egeth, 1994; Tipper, Weaver, Jerreat, \& Burak, 1994; Umilta, Castiello, Fontana, \& Vestri, 1995).

Support for both spatial- and object-based frames of reference have also been obtained from studies of patients with unilateral neglect caused by brain lesions. These individuals neglect information presented in the space contralateral to the side of the lesion, such that, for example, stimuli on the left side of space are neglected following lesions to the right parietal lobe. Information on the left side of an object, however, may also be neglected by these patients (Behrmann \& Moscovitch, 1994; Buxbaum, Coslett, Montgomery, \& Farah, 1996; Caramazza \& Hillis, 1990; Caramazza \& Hillis, 1990a; Driver, Baylis, Goodrich, \& Rafal, 1994; Driver \& Halligan, 1991;
Hillis \& Caramazza, 1995). For example, Behrmann and Tipper (1994) and Tipper and Behrmann (1996) demonstrated that, after initially viewing an object (a barbell), which then rotated slowly for 180 degrees, the neglect associated with the left side of the object accompanied the object to its new location. This was revealed by impaired detection of targets that appeared on the left of the object which now occupied a position on the right side of space, and improved target detection on the right of the object which was now on the left side of the space. Importantly, this left/right spatial reversal of neglect was only observed when the left and right sides of the object were explicitly connected by a bar such that one coherent object was perceived. When two separate circles were presented on opposite sides of the screen, rotation had little effect on neglect, and the neglect remained on the left side of space. Note 1 Importantly, although performance to left-sided targets was facilitated with object rotation, in some subjects, target detection on the left was still generally worse than detection on the right/ipsilesional side. An inference that could be drawn is that the simultaneous presence of both location- and objectcentered neglect yields the overall slower performance on the left which is

\footnotetext{
Note ${ }^{1}$ It is this latter result that motivates our use of object-centered and location/space-based frames of reference. Other reference frames have been described. For instance, scene-based (Driver \& Baylis, 1993) or between-object frames (Humphreys \& Riddoch, 1995) describe the relationships between different objects. Because neglect was unaffacted by the rotation of the unconnected objects in a scene-based frame, it was suggested that neglect was determined simply by spatial coordinates. Only when a single object was visible did neglect move with the rotation object, supporting the notion of an object-centered frame.
} 
then modulated by the object representation in the moving condition. In these data, the existence of two frames of reference is not directly observed, but rather is indirectly inferred as a way of explaining the interaction in the complex pattern of data.

More direct evidence for the simultaneous operation of two frames of reference comes from studies by Humphreys and Riddoch (1994, 1995). They described a patient who produces neglect explicitly in both spatial and object-based co-ordinates, depending on the task. When identifying individual letters of a four letter word, the patient neglected the right side, but when naming the word, he neglected its left side. Because this patient had lesions to both the right frontal and left parietal/temporal regions, it is reasonable to surmise that these neural structures mediated the left object-based and right space-based neglect, respectively (for similar findings with normal and brain-damaged subjects and a similar theoretical proposal, see, Egly et al., 1994; Egly, Rafal, Driver, \& Starrveld, 1994). Other work however, has shown neglect both for stimuli on the left and on the right even in patients with a unilateral lesion (Costello \& Warrington, 1987; Cubelli, Nichelli, Bonito, Tanti, \& Inzhagi, 1991; Riddoch, Humphreys, Luckhurst, Burroughs, \& Bateman, 1995). This observation is crucial for our current purposes because it demonstrates that a bilateral lesion is not necessary to produce neglect concurrently in two frames of reference.

An important feature of these neglect studies is that the two frames of reference in which neglect is observed are either inferred indirectly via the interpretation of complex interactions in the data pattern or are largely determined by task demands. In the latter case, object-based neglect is observed when subjects engage attention upon a single object, and location-based neglect is obtained when the task requires attention to move between objects. However, what has not been demonstrated is whether both spatial and object-centered neglect can be directly observed simultaneously in the same task in patients with unilateral lesions. If this were so, it would provide support for the view that spatial information is coded in more than one reference frame, and that, following brain damage, the attentional deficit may manifest in these different representations simultaneously.

The present investigation is based on the Behrmann and Tipper (1994) and Tipper and Behrmann (1996) findings. In the first instance, the left/right modulation of neglect by object-centered representations is replicated when the left and right circles of the barbell is probed. In addition, a further manipulation is adopted in which targets can appear on static square objects that remain stationary on the left and right sides of the display (see Figure 1). These static objects provide a stable background, demarcating the left and right of a location-based frame of reference. If both spatial- and objectcentered neglect can be observed simultaneously, then we predict that the left of the static object will be neglected while the left of the moving barbell object will be neglected. In this latter case this means that, after 180 degrees of rotation of the barbell, detection of targets will be worse on the right of the space. Thus, in terms of the side of space, we predict the following counterintuitive results: Targets that appear on the left of the static square stimuli will be poorly detected but targets will be much better detected in the circle on the right of the rotating barbell stimulus whose final locus is on 
the left, just adjacent to the static square stimulus (i.e., the facilitation afforded by the object-centered representation). In contrast, detection will be relatively good on the static square stimulus on the right side of space, and relatively poor in the left circle of the rotating object whose final resting locus is also on the right side of space (i.e. inhibition afforded by the object-centered representation). Hence detection of targets that are spatially adjacent in the same hemifield will vary because they are represented and neglected in different frames of reference (locationand

\section{$\underline{\text { A. Static }}$}

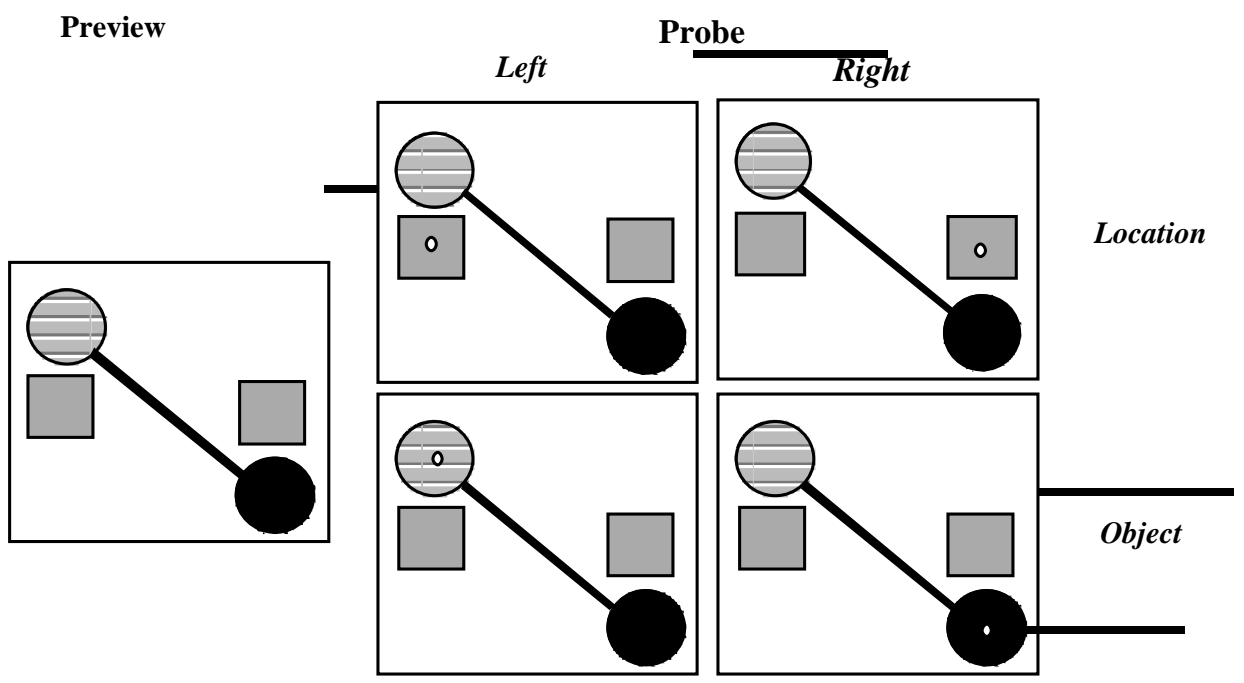

\section{B. Moving}

Preview

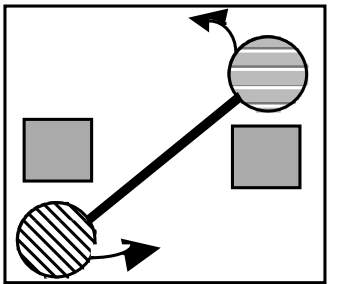

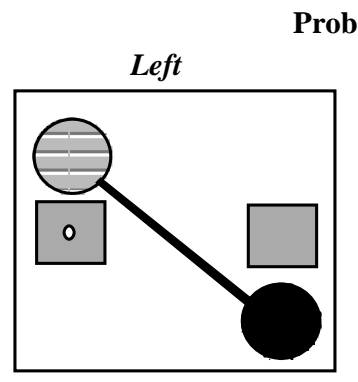

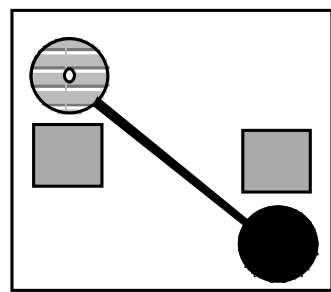

Probe

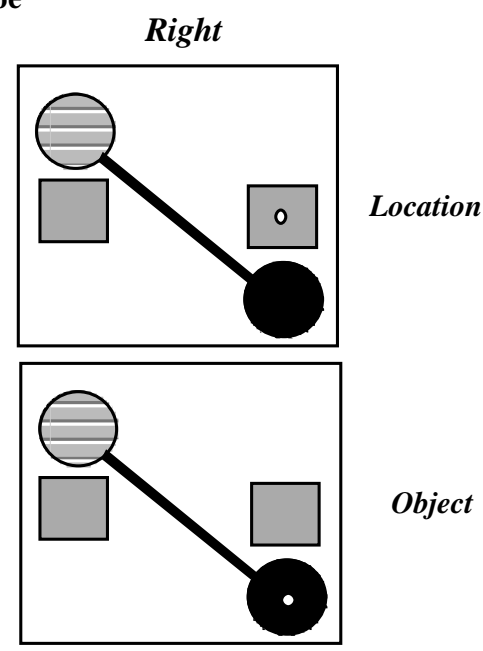

Figure 1: Schematic representation of (A) static and (B) moving display conditions with target probe shown in both the squares and circles as a function of side of space. 
Although we have explicitly defined what constitutes an object-centered frame in this particular paradigm in that the midline of the barbell determines what is to its right and left, exactly what constitutes the alternative, "location" reference frame is somewhat more ambiguous. In the context of this paradigm, for most of the patients for whom the visual presentation is foveal, and the midline of the viewer is aligned with the midline of the display, the location-based frame could refer to a set of allocentric coordinates centered either on the scene or on the environment (Kahneman \& Treisman, 1984; Treisman, 1992) or may even refer to what some have called a 'stimuluscentered frame' (Hillis \& Caramazza, 1995). Alternatively, left and right can be defined with respect to a set of egocentric coordinates centered on the viewer, with the midline determined by the axis of the eye, head or trunk (Karnath, Schenkel, \& Fisher, 1991). Given that both the scene-based and viewer-based frames are clearly important in influencing behavior (Bisiach, Capitani, \& Porta, 1985; Farah, Brunn, Wong, Wallace, \& Carpenter, 1990; Làdavas, 1987), distinguishing the individual contribution of these two reference frames is certainly worthy of future work. For the present purposes, however, our goal is to determine whether neglect can co-occur in multiple reference frames, one of which is objectcentered.

\section{Experiment 1}

Our purpose in this first experiment is to examine whether spatial information can be represented in more than one frame of reference simultaneously. If this were the case, responses to targets on the contralesional side defined with respect to each of the two different frames will be impaired in patients with neglect. Specifically, this would mean that targets on the left of the location-based frame would be more poorly detected than those on the right. Concurrently, targets on the left of the object-based frame, which now fall on the right side of space, will be more poorly detected than those on the right, which fall on the left side of space.

\section{Method}

Observers. The experimental group consisted of eight patients with left unilateral visual neglect sustained following a right hemisphere lesion. While right-sided neglect can occur following left hemisphere lesions, it is less common, less severe and less longlasting (Bisiach \& Vallar, 1988); because all of our subjects showed leftsided neglect, we will refer to the left as the contralateral side throughout this paper. All patients consented to participate. Two subjects were excluded from the sample because they failed to show the crucial object-based effect i.e., the interaction between the moving and static barbell condition and the side of space on which the target appeared Note 2 - Evidence of this pattern was a necessary precondition for subject inclusion to enable the investigation of

\footnotetext{
Note 2 We have noted in our investigations that approximately $20 \%$ of the patients do not show the object-centered effect. Exactly why this is so remains unclear to us and is the subject of current research. It is possible that the objectbased spatial representation is somewhat weaker or less dominant. Alternatively, it may be that not every subject shows object-centered neglect because there is additional support from the intact ventral system for this type of representation, making it more resistant to the effects of brain damage.
} 
object-based neglect along with other forms of neglect.

The remaining six neuropsychological subjects (one of whom, RH, had participated in one of our previous studies) were all righthanded and had lesions that involved the right parietal lobe although, as is usually the case, the lesion implicated a number of adjacent areas for many of the subjects. Although CT scan and radiology reports were obtained for all patients to determine the site of the lesion, it was not possible to obtain the images in all cases. Figure 2 shows the CT scans for two of the patients, RB [patient 3; panel A (i) and (ii)] and JT [patient 5; panel B (i) and (ii)], although these lesions sites are fairly representative of the lesions for some of the other patients too. RB suffered a post-operative right parieto-occipital haemorrhage following resection of a right parietal-occipital arterio-venous malformation. The scans show both the haemorrhage as well as the presence of surgical clips and an overlying craniotomy defect. JT suffered a right temporo-parietal middle cerebral artery infarction following the clipping of an aneurysm and evidence of the infarction and surgical clip is seen on the CT scans.

Figure 2: Two slices from CT scans obtained for patients RB (P3) and JT (P5). a) RB's scans reveal a postoperative right parieto-occipital haemorrhage and an overlying craniotomy defect. b) JT's scans reveal a right temporo-parietal middle cerebral artery infarction. Surgical clips are evident in both patients' scans.
Two of the patients, p4 and p5, had visual field defects and for them, the displays were presented entirely in their intact visual field. Because neglect is not a sensory deficit, even when information is presented solely in the intact visual field, information on the relative left is processed less well than that on the right (D'Erme, Robertson, Bartolomeo, Daniele, \& Gainotti, 1992; Làdavas, Petronio, \& Umilta, 1990).

The diagnosis of neglect was made on the basis of a standardized battery of bedside examinations which includes spontaneous drawing of a clock and a daisy, a line cancellation task (modified Albert's line cancellation task, Albert, 1973), figure cancellation test, the Bells test (Gauthier, Dehaut, \& Joanette, 1989) and a line bisection task. A score was assigned for each subtest reflecting the degree of neglect, relative to the performance of a group of age-matched normal control subjects. A total neglect score, cumulative across all the screening tests, was then calculated (Black et al., 1994; Black, Vu, Martin, \& Szalai, 1990). The cumulative maximum neglect score based on these four tests was 100 with a score of 6 or greater being classified as neglect, and higher scores denoting increased severity: scores over 75 indicate severe neglect, scores below 30 indicate mild neglect and those in between indicate moderate neglect. All patients obtained a score greater than 25 . Table 1 presents the biographical, lesion and neglect scores for the 6 patients. Examples of the left-sided neglect performance of some of the patients on a variety of these neglect screening tests is presented in Figure

3.

Table 1

Biographical, lesion and neglect data for the 6 experimental subjects. 


\begin{tabular}{lcccccc}
\hline Patients & $\mathbf{1}$ & $\mathbf{2}$ & $\mathbf{3}$ & $\mathbf{4}$ & $\mathbf{5}$ & $\mathbf{6}$ \\
\hline Initials & $\mathrm{RH}$ & $\mathrm{VD}$ & $\mathrm{RB}$ & $\mathrm{JB}$ & $\mathrm{JT}$ & (K) \\
Age & 63 & 71 & 64 & 65 & 58 & 81 \\
Gender & $\mathrm{F}$ & $\mathrm{F}$ & $\mathrm{M}$ & $\mathrm{M}$ & $\mathrm{M}$ & $\mathrm{M}$ \\
Yrs. education & 10 & 9 & $?$ & 12 & 10 & 8 \\
Months post-onset & 20 & 4 & 31 & 2 & 3 & 14 \\
CT lesion site & PTO & $\mathrm{P}$ & $\mathrm{PO}$ & $\mathrm{PO}$ & $\mathrm{PT}$ & $\mathrm{PT}$ \\
Lesion type & Aneurysm & Infarct & Aneurysm & Infarct & Aneurysm & Infa1 \\
Neglect scores: & & & & & & \\
Line cancel (left/right) & $0 / 0$ & $0 / 0$ & $7 / 3$ & $8 / 0$ & $3 / 0$ & $12 / 1$ \\
Figure cancel (left/right) & $6 / 0$ & $11 / 0$ & $9 / 1$ & $12 / 0$ & $23 / 0$ & $19 / 1$ \\
Bells test (left/center/right) & $8 / 0 / 1$ & $5 / 4 / 2$ & $11 / 1 / 1$ & $11 / 1 / 1$ & $?$ & $?$ \\
Line bisection (\% deviation) & 31 & 6 & 6 & 8 & 0 & 8 \\
\hline TOTAL NEGLECT SCORE & 29 & 37 & 51 & 62 & $?$ & $?$ \\
\hline
\end{tabular}

${ }^{+} \mathrm{F}=$ frontal, $\mathrm{P}=$ parietal, $\mathrm{T}=$ temporal, $\mathrm{O}=$ occipital

* JT and IK did not complete the Bells test and so no score on that test nor a cumulative neglect score could be calculated.

Figure 3: Examples of neglect behavior on a subset of screening tests used to diagnose neglect: (a) copy daisy - patient JB; (b) copy clock - three consecutive attempts labeled 1 through 3 by patient VD; (c) line cancellation - patient RB. It is interesting to note the multiple cancellation of lines on the ipsilesional right, and consistent with the strong attraction of ipsilesional items; (d) Bells test (Gauthier et al., 1989) - patient IK. Note the omission of contralesional left information in all cases.

A control group, consisting of six righthanded elderly control subjects all of whom consented to participate, was recruited from the community through an outreach program run by the Academy for Lifelong Learning at Carnegie Mellon University. Subjects were matched pairwise to the six patients in age and gender. All control subjects were right-handed, none had a previous history of neurological disease and none scored more than 8 on the diagnostic tests of neglect. The mean age of the subjects was 65 (range 58-69), not significantly different from that of the patients, $F(1,10)=1.7$, n.s.

Stimuli. Two circles, $2.1 \mathrm{~cm}$ in diameter and subtending a visual angle of $3^{\circ}$, drawn with a black border, one colored blue and one colored red, appeared on a computer screen. The two different colors were necessary to disambiguate the sides of the object and the side of the blue and red circle was consistent for each subject throughout the testing sessions, and counterbalanced across subjects. The distance between the nearest inner edges of the circles was $7.2 \mathrm{~cm}\left(10.3^{\circ}\right)$. The length of the entire barbell was $11.4 \mathrm{~cm}$ $\left(16.2^{\circ}\right)$. In addition to this barbell, two gray squares, $2.1 \mathrm{~cm}^{2}$ and subtending $3^{\circ}$ of visual angle, were placed along the horizontal midline of the display, as depicted in Figure 1. The distance between the edges of the squares was 5.5 $\mathrm{cm}\left(7.9^{\circ}\right)$. The target, a single white circle, was $.7 \mathrm{~cm}$ in diameter $\left(1^{\circ}\right)$. 
Procedure. Stimulus presentation and response recording were controlled by a Macintosh Powerbook 540C with a built-in 193 x $145 \mathrm{~mm}$ screen and $640 \times 480$ resolution. Subjects were seated approximately $40 \mathrm{~cm}$ from the screen. The phrase "Press start key" appeared in the center of the screen before each trial. The experimenter pressed the key when the subject was ready and, immediately thereafter, the display appeared on the screen. The joint barbell-square displays appeared in two conditions, when the barbell was either static or moving. The squares remained static in both conditions.

In the static condition (see Figure 1A), the preview display was presented, remained stationary for $2694 \mathrm{~ms}$ and then, on two-thirds of the trials, the white probe appeared in either the left or right circle of the barbell or the left or right of the square, all with equal probability but randomly ordered (the target-present trials). The target and display remained on the screen together until a key was pressed or for an additional $3 \mathrm{~s}$ if there was no response. On the remaining one-third of the trials, no target appeared and the display remained on the screen for a further $3 \mathrm{~s}$ before the trial was terminated (the target-absent trials). Subjects were instructed to press a single, centrally placed key on a button-box as quickly and accurately as possible when they detected the presence of the target. They were not to respond on target-absent trials. Subjects responded with their dominant right hand. Reaction time (RT) and accuracy to detect the target were measured. Omission and commission errors were noted and feedback, consisting of an auditory tone, was provided to the subject on each trial when an error of either kind occurred.

In the moving condition (see Figure 1B), the preview display appeared, remained stationary for $1 \mathrm{~s}$ with the barbell displaced $57.5^{\circ}$ from horizontal. The barbell then underwent a $115^{\circ}$ rotation (pivoting on the center of the bar), traversing 14 intermediate positions (15 'jumps' of $7.66^{\circ}$ each) and giving rise to the perception of apparent motion. Each position was held for 121 ms duration, for a total rotation time of $1694 \mathrm{~ms}$. The total time prior to the appearance of the target was equivalent to that of the static condition. The direction of rotation was randomized, with an equal probability of clockwise (CW) and counterclockwise (CCW) rotation. When the stimulus had completed the rotation and reached its 'end state', on the target-present trials which constituted two-thirds of the trials, the target probe appeared randomly but with equal probability in the left or right circle (now left circle on ipsilateral right side and right circle on contralateral left side) or left or right square. The target remained on the screen until a response was made or until a further $3 \mathrm{~s}$ had elapsed. On the remaining, target-absent trials, the display remained on the screen for a further $3 \mathrm{~s}$ and then the trial was terminated. As is evident from Figure 1, the display in which the target probe finally appeared was identical in the static and moving condition. In the moving condition, however, because of the rotation, the left of the barbell was on the right of space and the right of the barbell on the left of space. A comparison between the static and moving condition, therefore, allows one to determine the contribution of the object frame to detection time in these patients with neglect. Instructions for responding and feedback were identical to those in the static condition and RT and accuracy of target detection were measured. 
Design. The design of the experiment was $2 \times 2 \times 2 \times 2$ with group (control, neglect) as a between-subject factor and position of target (in circles of barbell circles/object or in squares/location), side of space (left, right) and condition (moving or static) as within-subject factors. Subjects performed 8 blocks of 60 trials, four in the static and four in the moving condition, for a total of 480 trials with block order counterbalanced across subjects. Within each block, there were forty target-present trials, with an equal crossing of side of space of target and shape of display (circles versus squares). Subjects were given a break between blocks and practice trials were given before the first block of each of the static and moving stimuli. RT analyses were performed only on correct, targetpresent trials. For the patients, RTs which exceeded the mean by two standard deviations were removed. The median RT was used for the normal subjects. All post hoc testing was done using Tukey HSD tests with $p<.05$. A comparison of the RTs in the moving condition for clockwise compared with counterclockwise trials was conducted first. Because direction of rotation in the moving condition did not influence RTs significantly (as was also the case in our previous studies), the remaining analysis is collapsed across rotation direction.

\section{Results}

The central question addressed by these data is whether neglect can occur for the left in location-based coordinates (left versus right square) concurrently with neglect in objectbased co-ordinates (left versus right circle of barbell). An ANOVA with one between-subject variable (group) and three within-subject variables [condition (static, moving), frame of display (square, circle) and target side (left, right)] was performed on the RT correct data. The findings revealed a significant four-way interaction indicating a difference in the performance of the two groups $\quad(F(1,10)=9.04, \quad M S E=3817.6$, $p<.05)$. To highlight these differences in performance across the groups, we describe the data for the two groups separately, and plot the findings for each in Figure 4. The error rates were $1.3 \%$ and $2.8 \%$ for the normal and neglect group, respectively, and are too low to be subjected to statistical analysis. This very low error rate is not surprising given that exposure duration was sufficiently long for subjects to perform this very simple target detection task. 


\section{Circle (object coordinates) Square (location coordinates)}

A. Control subjects

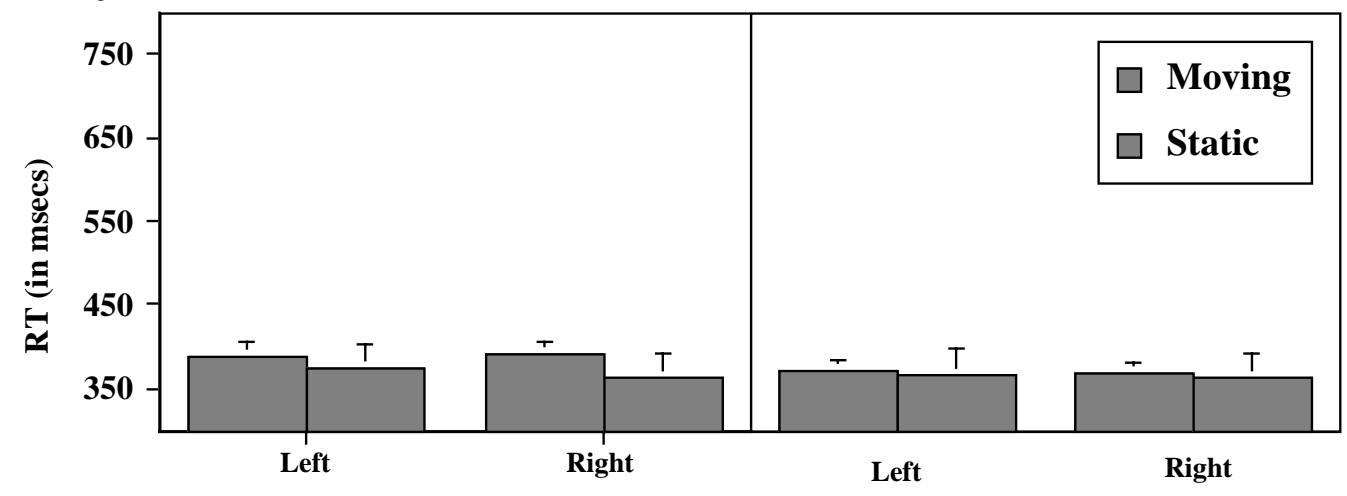

B. Neglect subjects

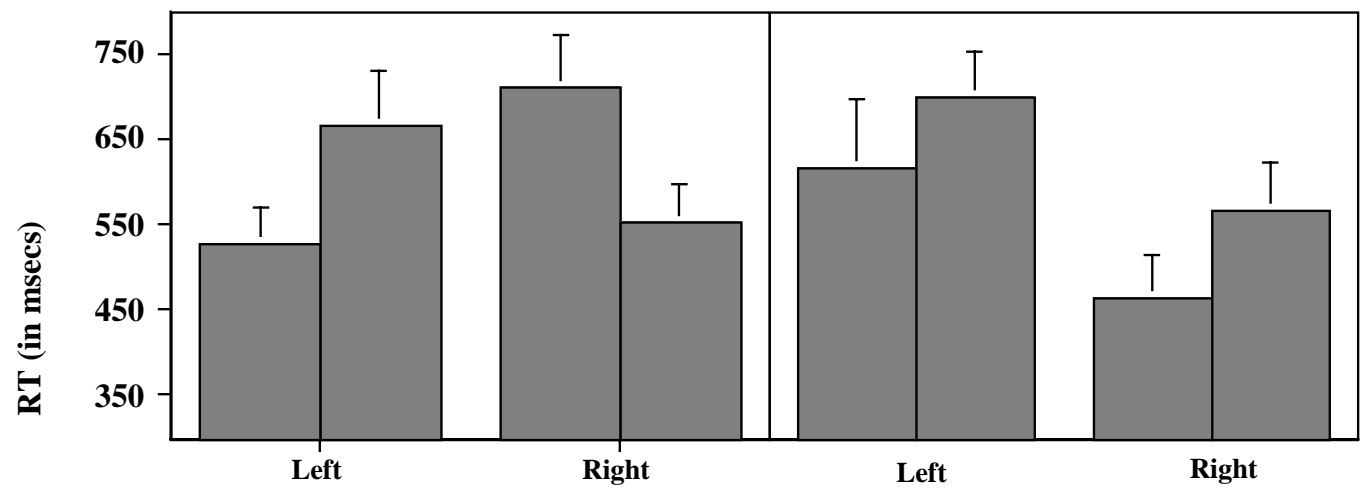

Side of target

Figure 4: Mean of median reaction time and standard errors for (A) normal control subjects and (B) mean of mean reaction time for neglect patients for targets on the left and right in object- and location-coordinates as a function of condition (static and moving).

Normal subjects were able to detect the target $9 \mathrm{~ms}$ faster when it appeared in the squares than when it appeared in the circles, $\quad(F(1,5)=8.5, \quad M S E=127.1$, $p<.05)$, with a difference of $18 \mathrm{~ms}$ between the moving circle and moving square conditions and no difference between the static circle and static square conditions, $(F(1,5)=9.7$, $M S E=80.02, p<.05)$. No other effects were significant. There is no obvious interpretation for the slight advantage for the squares over the circles and no obvious reason why this is especially so when the circles of the barbell rotate. Of note here and perhaps most important for the present investigation is that the normal subjects demonstrate equivalent detection times for left- and right- sided events and do not exhibit an asymmetry for the side on which the target appears, nor does side interact with the frame of the display (circle or square). These findings suggest that there are no fundamental biases with regard to side which might be important when interpreting the findings from the neglect subjects. 
For the neglect participants, the major finding was that the three variables, frame of display (square, circle), side of space of target (left/right) and condition (static/moving) affected detection time interactively, $(F(1,5)=9.66, \quad M S E=7551.5, \quad p<.05)$. Importantly, as a group, the neglect subjects showed the significant objectcentered effect, manifest as an interaction between condition and the side of space for targets in the circles, $(F(1,5)=38.8, \quad M S E=1421.8, \quad p<.005)$. Relative to the static barbell, in the moving condition, subjects are, on average, $138 \mathrm{~ms}$ faster to detect the left target and $155 \mathrm{~ms}$ slower to detect the right target (see left panel in Figure 4B). This significant left-sided facilitation and significant right-sided inhibition replicate the previously established object-centered effect (Behrmann \& Tipper, 1994; Tipper \& Behrmann, 1996). This result is not surprising, however, given that neglect subjects were selected for inclusion into this sample only if they showed either the left-facilitation or right-inhibition in their individual data.

The pattern of data is quite different for the squares compared with the circles; detection was $141 \mathrm{~ms}$ poorer for targets on the left of the display than on the right and this left-sided inferiority held regardless of whether the barbell moved or remained static (see right panel in Figure 4B). There is also a joint effect on RT of frame of display with the side of space on which the target appears, $\quad(F(1,5)=65.8, \quad M S E=1415.3$, $p<.005)$; collapsed across condition, RTs to targets on left squares are $51 \mathrm{~ms}$ slower than to targets on right squares but the converse is true in the case of the circles (left $36 \mathrm{~ms}$ faster than right) because of the left facilitation and right inhibition associated with the barbell. Finally, there is a significant joint effect of side of target and moving/static condition on RT, $(F(1,5)=38.8$, $M S E=1421.8, \quad p<.005)$, such that, collapsed across squares and circles, left static trials were, on average, $121 \mathrm{~ms}$ slower than right static trials, but the difference between left and right moving trials was $15 \mathrm{~ms}$ with the slight advantage for the left trials.

This pattern of data, including the reversal of the left inferiority on the static barbell when the barbell rotates, and the persistent left inferiority in the squares, irrespective of whether the barbell rotates or remains static, is even manifest in the data from the individual subjects. Figure 5 shows the RT data as a function of condition for the circles and the squares for each of the six subjects separately. The left-sided facilitation for the moving over the static circle conditions is seen in 4 of the 6 subjects (not significant in $\mathrm{P} 2$ or $\mathrm{P} 4$ ) and is most clearly evident in P1. The rightsided inhibition for the moving over static circle conditions is observed in 5 out of 6 subjects (not in P1) although the effect is only marginally significant in $\mathrm{P} 2$. In all cases, detection of targets on the left of the squares was poorer than on the right and there was an advantage for the moving over the static displays in all but the left trials for P1 and P5 but no interaction between side of target and moving/static condition. Importantly, then, the major finding of a cooccurrence of object-based neglect with neglect in a second set of coordinates defined by the squares, was upheld in the patterns of the individual data albeit to varying degrees in the different subjects. 


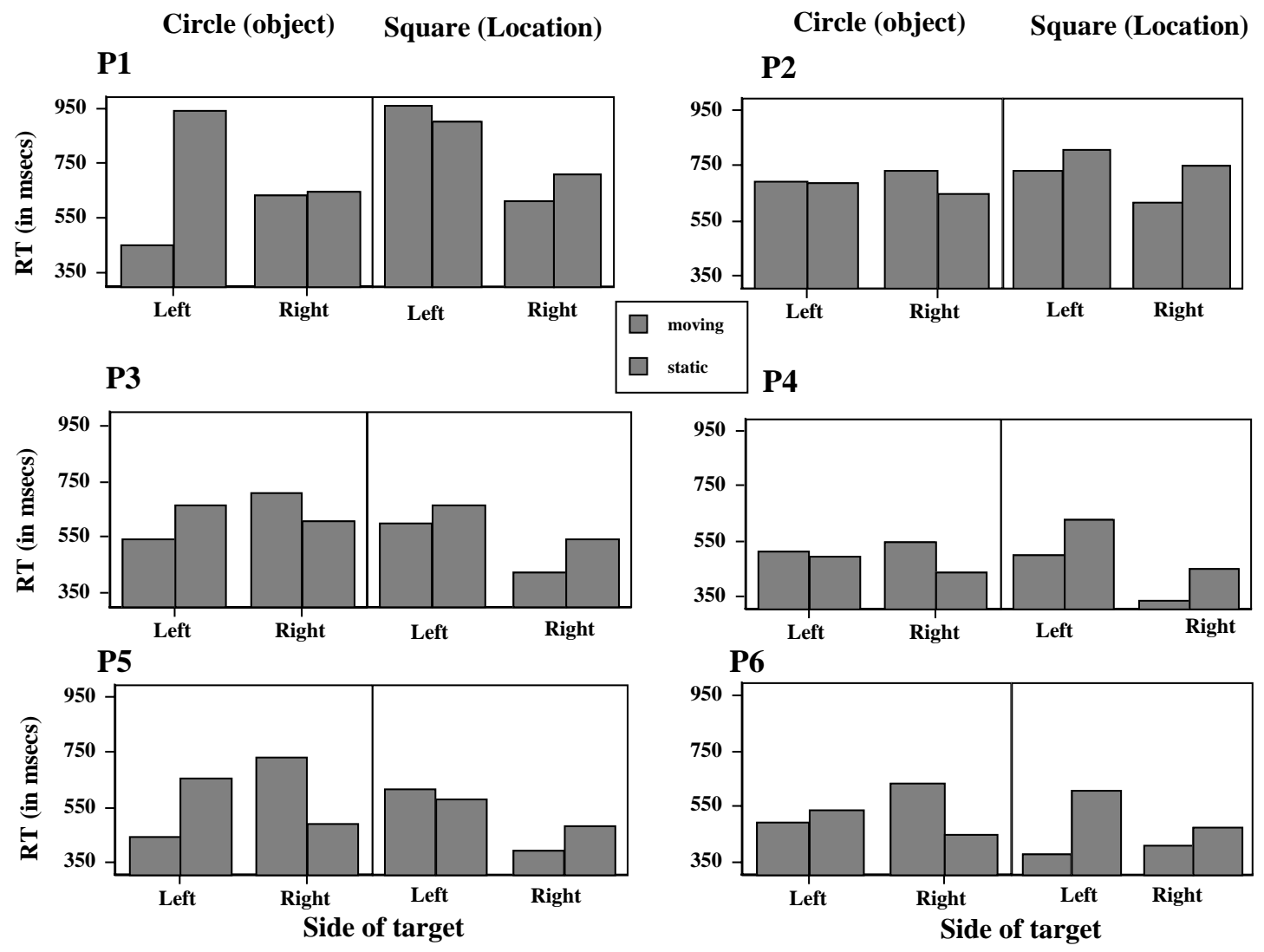

Figure 5: Mean reaction time for targets on the left and right in object- and locationcoordinates as a function of condition, plotted for the six patients individually.

\section{Discussion.}

The first major question addressed in this study concerned the presence of neglect in both objectcentered and location-based frames simultaneously. The key finding from this study was that in a group of neuropsychological subjects in whom object-centered neglect was observed, manifest as left-side facilitation and right-sided inhibition for moving over static displays, we also observed poorer performance for targets on the left than the right, defined in location-based coordinates. Thus, in a situation in which the left and right sides of two different co-ordinate frames were probed in a mixed block of trials, performance for targets on the contralesional side defined with respect to two different reference frames was noted simultaneously. Under these experimental conditions, the subjects could not develop any obvious expectations or contingencies about which reference frame might be probed and neglect was evident in both. Importantly, the co-occurrence of neglect in two reference frames cannot be attributed to two different anatomical locations as these patients all had a single right-hemisphere lesion. Instead, these data suggest that a unitary spatial attentional mechanism, mediated by the right hemisphere, can select and access information represented within more than one spatial coordinate system.

\section{Experiment 2}


Having established the coexistence of neglect in more than one spatial reference frame, a second question concerns the distribution of attention or the relative extent of neglect in these two frames. Interestingly, the degree of neglect (difference between mean RT on the left and right over the average of left and right) in Experiment 1 was roughly equivalent in the two reference frames in the moving condition with neglect severity of $29.2 \%$ and $27.7 \%$ in the object and location frame, respectively. What is unclear is whether attention is always equally distributed in these two frames or whether this is simply a response to the contingencies in the sampling probabilities. In the previous experiment, the probability of target occurrence is equally balanced in the location-based (squares) and objectcentered (barbell) frames and one might imagine that the relative weightings of the information in each frame is approximately equal.

To determine whether the distribution of attention or weighting between the frames can be altered so that neglect may be increased or decreased as a function of the target contingencies, in Experiment 2 we manipulate the probabilities of the probe in the two reference frames. For example, in some blocks of trials the target appears in the barbell $80 \%$ of the time and in the squares only $20 \%$ of the time. If attention is equally allocated between the two frames by default and this holds irrespective of the contingencies, then the target probability manipulation should have no effect on RTs. On the other hand, if neglect reflects a pathology of attention, and attention can be flexibly allocated according to the demands or contingencies of the task (Baylis, Driver, \& Rafal, 1993; Vecera \& Farah, 1994; Watson \& Kramer,
1998), then when attention is predominantly focused on one reference frame (e.g., object-centered), detection of targets on the left in this frame may differ compared with when this frame is probed less often. Similarly, detection of targets on the right may differ as the contingencies of the task favor a particular reference frame.

In some theoretical accounts, one might predict that as a particular reference frame is probed more often, more attention is directed to the stimuli represented in this frame, and detection will be facilitated even for items on the contralesional left side. Empirical data consistent with this particular conceptualization comes from the finding that, for example, in a covert attention cueing paradigm, target detection is facilitated if covert attention is precued to the target location i.e., attention enhances subsequent processing (Posner, 1980; Posner \& Cohen, 1984). From such a perspective, when the probability of sampling targets in a particular frame is increased and attention distributed more often in such a frame, then we might expect to see a decrease in neglect as the sampling probability is increased.

There is, however, an alternative conceptualization of attention and neglect that is growing in popularity. On this account, we might expect to see an increase in neglect severity as probability is increased. Such a prediction is compatible with a view of selective attention in which attention is conceptualized as a more dynamic, competitive mechanism. On such an account, objects compete with each other for selection and further processing and the object that eventually 'wins' is the attended stimulus (Desimone \& Duncan, 1995; Duncan, 1996; Duncan \& Humphreys, 1989). These views suggest that multiple 
objects in the visual input compete for representation, analysis and control and attention is an emergent property of many neural mechanisms working together to resolve this competition. Competition is considered a general principle of neural selection, working across many different areas of the brain (Humphreys, Romani, Olson, Riddoch, \& Duncan, 1994).

Application of this competition interpretation to the case of neglect is as follows: the brain-damage imposes an exaggerated negative bias on left-sided stimuli and these stimuli are always disadvantaged in a competition between potential left and right-sided targets. Within such a competitive framework, the effects of the probability manipulation may work in the following fashion. When one frame of reference is probed with increased frequency i.e. the gain on the spatial bias is exaggerated such that the salience of information on the ipsilesional right is enhanced and that on the contralesional left is reduced. This marked left-right difference yields very little competition and the right side easily wins out over the poorly detected left-sided targets, giving rise to slowed time to detect left targets and increased neglect. When the representation defined in a particular frame is probed less frequently, the left-right differential is not as strong and the left-sided target is detected somewhat faster.

\section{Method.}

The identical stimulus and displays used in Experiment 1 were also used in this experiment. The only two methodological differences between the two experiments were that, firstly, only the neglect patients but no normal subjects participated in this experiment and, secondly, that two further testing sessions took place for each neglect patient. The same six neuropsychological subjects who participated in Experiment 1 took part in this experiment. In these additional sessions, the weighting of a particular reference frame was manipulated withinsubject. In one session, the probability that the targets would appear in the object-centered frame was $80 \%$ and in the location-based frame was $20 \%$ and in the second session, these probabilities were reversed. For half the participants, the sessions proceeded in the order described above and for the remaining half, the order was reversed. Within each probability level, eight blocks of 60 trials were run for a total of 480 trials. At $80 \%$ probability, the number of target-present trials on each of the left and right was 128 and at $20 \%$ probability, there were 32 targets on each of the left and right, for a total of 320 target-present trials. The remaining 160 trials were target-absent. The participants were explicitly informed of the particular probabilities at the start of each session and a block of 60 practice trials was run with this contingency. The analysis procedure used was identical to that in Experiment 1.

\section{Results.}

If the demands of the task affect the salience or weighting assigned to a representation and the patients exploit these contingencies, then one might expect to see differing patterns of RT data reflecting the relative sampling probabilities in the two different frames of reference. As in Experiment 1, the error rates in Experiment 2 were extremely low, comprising fewer than $3 \%$ of the trials. The analysis of the RT data involved a repeated-measures analysis of variance on the correct target-present trials with proportion $(20 \%, 80 \%)$, frame of display (circle, 
square), condition (static, moving) and side of space (left, right) as withinsubject variables. RT values which exceeded 2 SDS from the mean of a given cell were rejected and RTs were collapsed across direction of rotation in the moving condition. Figure 6 shows the mean RT and standard error across subjects in the static and moving condition for targets appearing on the left and right as a function of sampling probability and as a function of whether the targets appear in the circles (object co-ordinates) or squares (location coordinates).

\section{A.Circles (object-centered)}

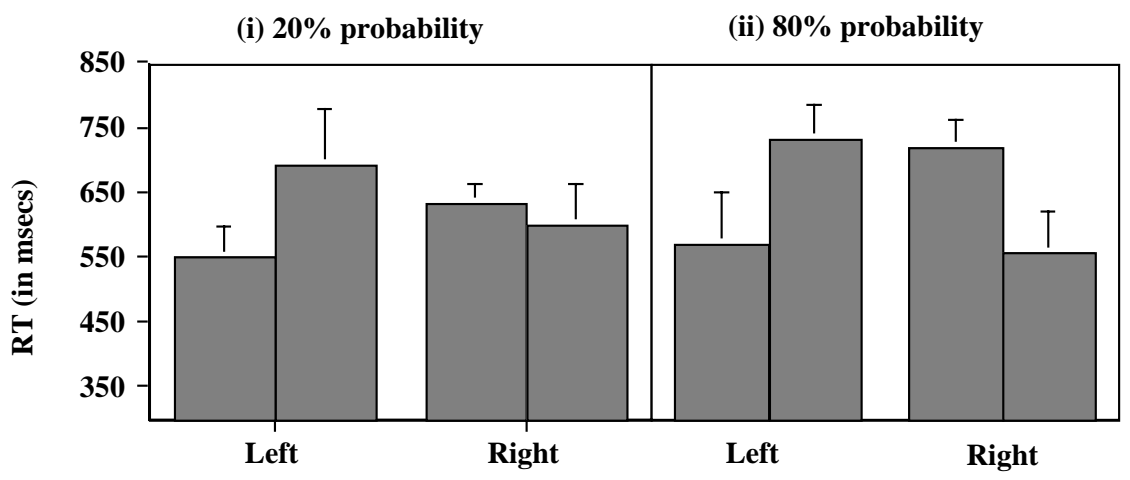

\section{B. Squares (location co-ordinates)}

(i) $20 \%$ probability

(ii) $80 \%$ probability

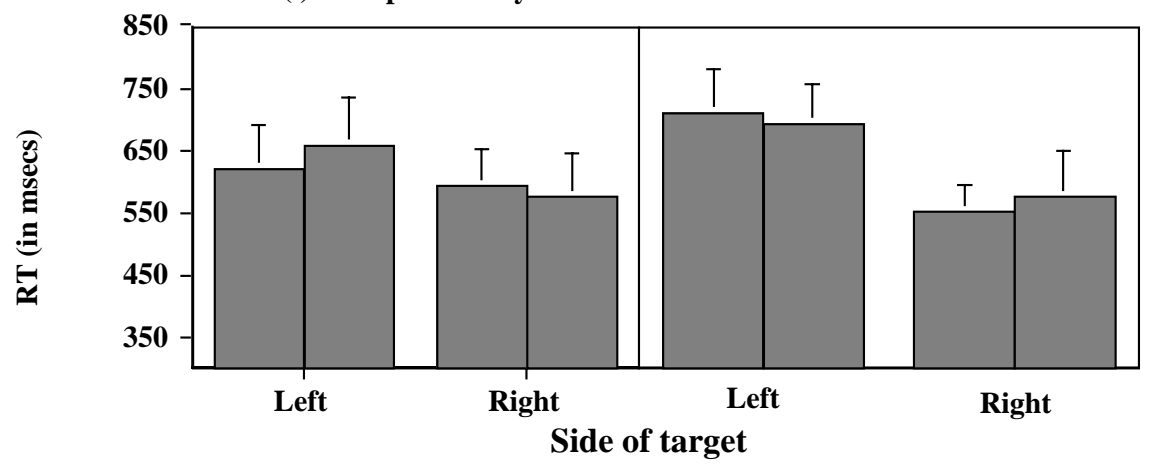

Figure 6: Mean of mean reaction time for neglect patients for targets on the left and right in (A) object- and (B) location-coordinates as a function of condition (static and moving) and sampling probability.

The most notable finding is that there is a four-way interaction, $(F(1,5)=8.2$, $\mathrm{MSE}=2892.7, p<.05)$. If we consider the pattern of data separately for the circles (panel A) and squares (panel B), the nature of the interaction becomes clear. As is evident from panel A of Figure 6, in both the $20 \%$ and $80 \%$ probability situations, the signature of objectcentered neglect is evident; that is, there is significant left-sided facilitation and right-sided inhibition in the moving relative to the static baseline. This replicates the pattern of data obtained in Experiment 1 (see Figures 4 and 5 for comparison). There is, however, an 
effect of the probability manipulation on the severity of object-centered neglect: although object-centered neglect is observed under both manipulations, the extent of the effect is more pronounced in the $80 \%$ than in the $20 \%$ condition. This increase in the object-centered effect comes about predominantly because of the increased inhibition for targets on the right in the moving condition relative to the static condition. Whereas the difference on the left between the static and moving circles in the $20 \%$ and $80 \%$ probability situations is $140 \mathrm{~ms}$ and $164 \mathrm{~ms}$, revealing a trend towards increased facilitation in the $80 \%$ condition, the difference is very marked for right-sided items. Inhibition on the right in the moving condition is only 33 $\mathrm{ms}$ in the $20 \%$ probability condition but is $166 \mathrm{~ms}$ in the $80 \%$ probability condition. These findings reflect an increase in the neglect pattern in objectcentered coordinates when this frame is probed more often but the increase is more pronounced for targets on the right.

Turning to the squares, we see location-based neglect in both the $20 \%$ and $80 \%$ probability situations, as reflected in the slowed RTs to left compared with right targets. Interestingly, whereas there is only a 55 ms difference between detection time for left and right targets in the 20\% condition (collapsed across static and moving conditions), subjects are $132 \mathrm{~ms}$ slower to detect left than right targets in the $80 \%$ condition, as is evident from panel $\mathrm{B}$ in Figure 6. This again reflects an increase in the extent of the neglect as the sampling probability increases.

These findings are summarized in Figure 7 which illustrates the increase in neglect in each of the two reference frames as the probability increases. To visualize the influence of target probability on neglect in the two reference frames we have replotted the data in terms of a difference score between RTs for targets on the left minus right (Y-axis) for static and moving displays separately. The $\mathrm{X}$-axis refers to the sampling probability. The effect in the circles or object coordinates (Panel A) is considered separately from the effects in the squares or location coordinates (Panel B). The error bars reflect the standard error of the differences across the subjects between RTs for left and right targets. 
A. Circles (object co-ordinates)

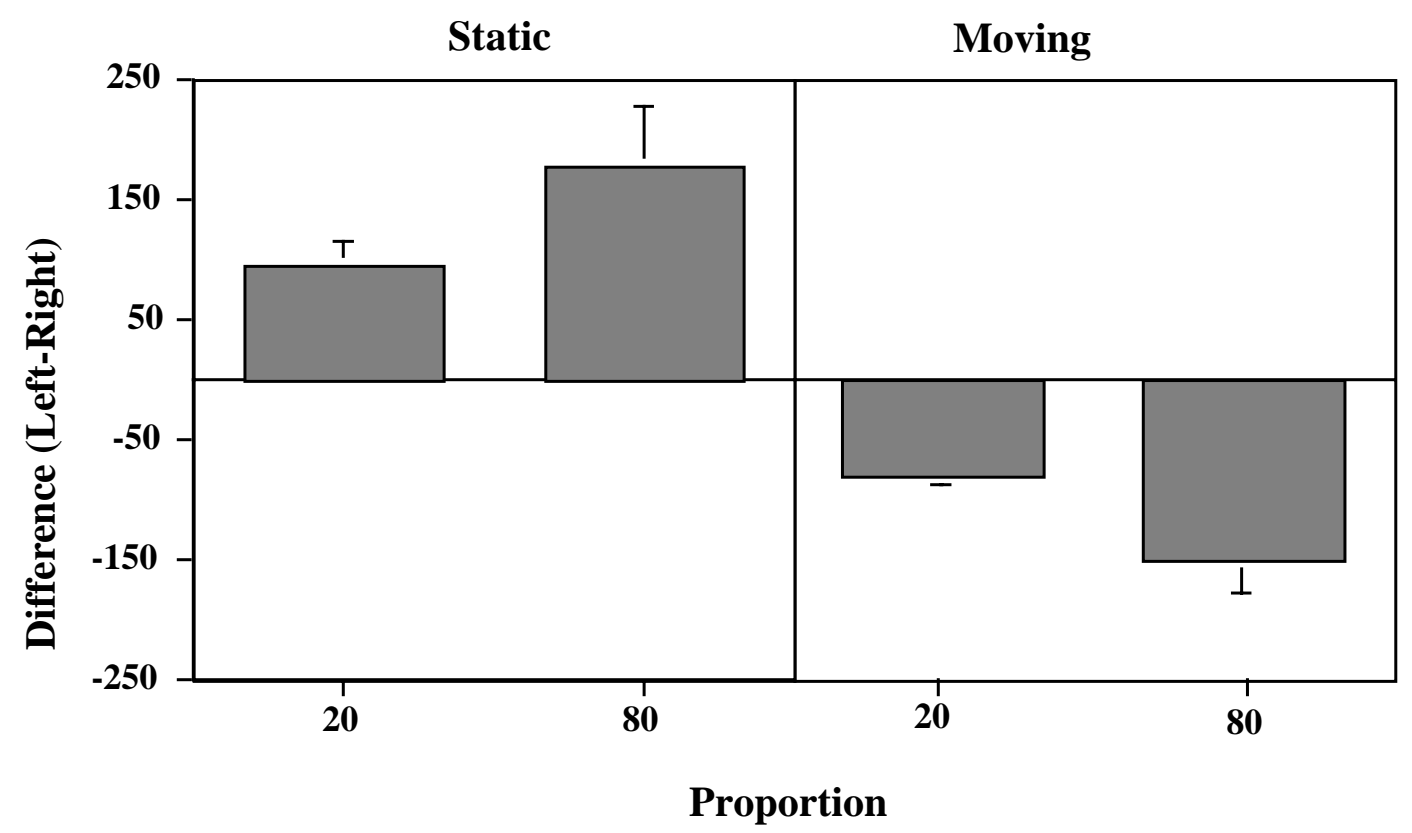

B. Squares (location co-ordinates)

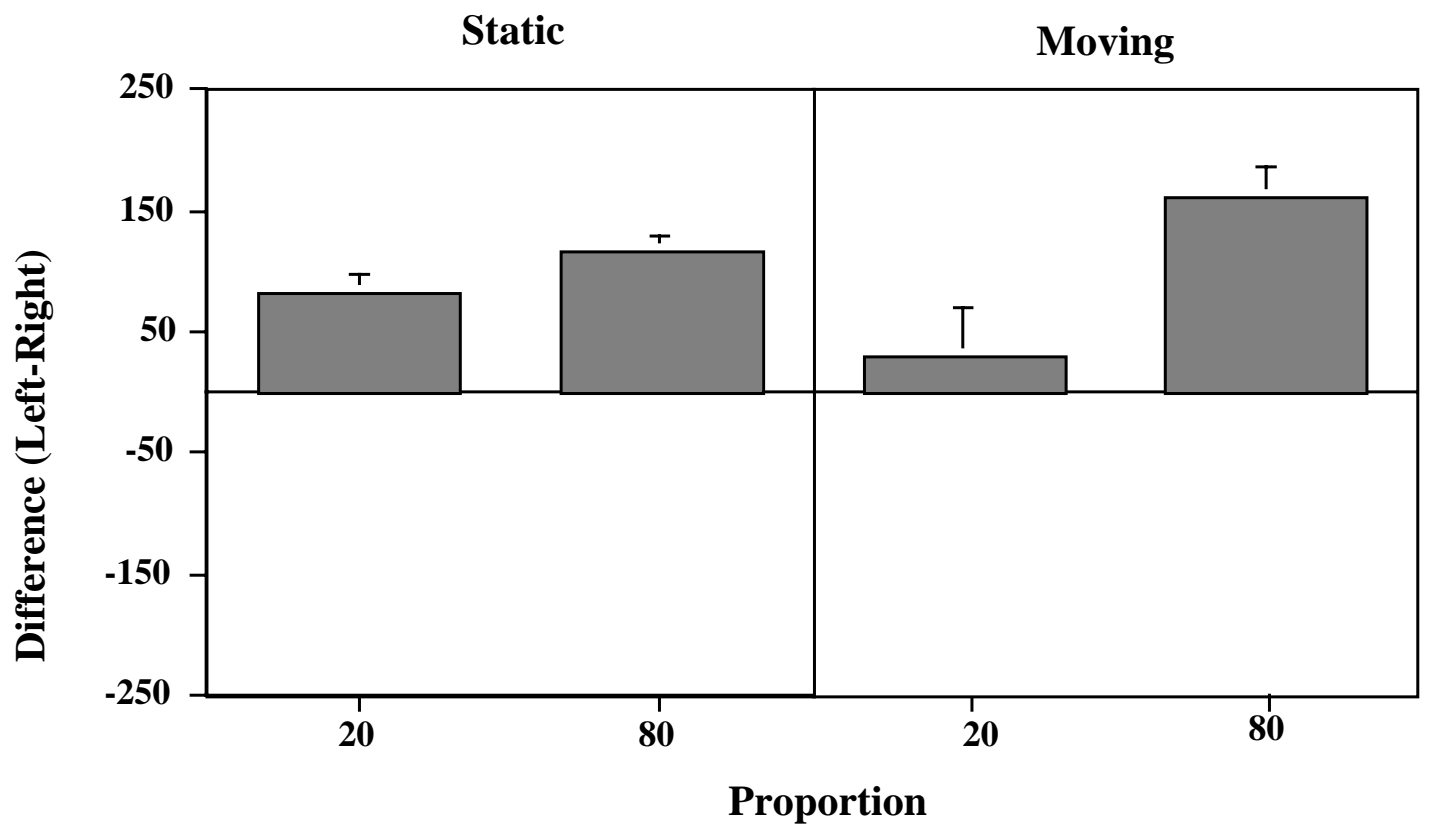

Figure 7: Difference (left minus right) in RT for targets appearing in (A) object- and (B) location-coordinates in the static and moving condition as a function of sampling probability.

If we consider the situation when the display is static, as is evident from the left sides of panel $\mathrm{A}$ and panel $\mathrm{B}$, the severity of neglect increases with 
probability of the target, but this is much more salient in the case of the circles of the barbell than in the case of the squares. Thus, as probability increases from $20 \%$ to $80 \%$, neglect increases from $82 \mathrm{~ms}$ to $116 \mathrm{~ms}$ in the squares, whereas neglect increases from $93 \mathrm{~ms}$ to $176 \mathrm{~ms}$ for the barbell circles. Even more striking, when the barbell rotates, we see a very marked effect of probability of sampling in both the squares and circles. For the circles, neglect increases in the object-centered frame as the sampling probability increases and because object-centered neglect is reflected as a slowing in rightsided RT and speed-up in left-sided RTs, we see negative difference scores between the left and right in panel A of Figure 7. Whereas the difference in RTs for the left over the right was $-81 \mathrm{~ms}$ on average in the $20 \%$ condition, it was $152 \mathrm{~ms}$ in the $80 \%$ condition. For the squares, neglect increases from $29 \mathrm{~ms}$ to $159 \mathrm{~ms}$ in the location-based frame as sampling increasing from $20 \%$ to $80 \%$ probability, as seen in the right display of panel B. Taken together, these findings indicate an exaggeration of the neglect effects as the weighting of a particular frame is increased.

\section{Discussion.}

There are two major results that emerge from this experiment. The first finding is that neglect can occur in both locationbased and object-centered representations concurrently, thus replicating the results obtained in Experiment 1. The second and novel finding is that there is a modulation of the severity of neglect when the sampling probabilities of targets appearing within the location-based or object-centered representations are systematically varied. Interestingly, and perhaps counterintuitively, as the targets in one frame are probed with higher probability, the extent of the neglect increases in that frame. Thus, these findings go further than demonstrating the co-existence of neglect in more than one coordinate system by showing that the contingencies of the task can alter the severity of the neglect. As the sampling probabilities are altered, so attention can be flexibly and strategically allocated between reference frames to accommodate the behavioral demands of the task.

A particularly interesting aspect of the finding is that the facilitation and inhibition associated with the circles appears to have no obvious effect on the squares; detection of left and right targets in the squares is unaffected by the barbell rotation. Thus, attentional resources allocated to the barbell prior to its rotation provide neither a benefit for the left square (when detection in the circle is facilitated relative to the static circle) nor a disadvantage for the right square (when detection in the circle is inhibited relative to the static circle). Even in the high-probability sampling trials in the circle in which we see the maximum effect of the rotation, there is still no significant difference between the squares when the barbell rotates compared with when it remains stationary. Thus, despite the physical proximity of the square and circle, the costs and benefits associated with the circle do not transfer to the square. These findings have two important implications: first, there appears to be no transfer of the spatial biases between the squares and circles, suggesting that the different frames of reference are independent. The second important implication is that the well-established attentional facilitation one sees for stimuli located near a target (Eriksen \& Hoffman, 1972; Hoffman \& Nelson, 1981; Tsal \& Lavie, 1988) is based not 
solely on the proximity, defined by physical distance between stimuli on the screen, but on whether or not the stimuli both appear within the same reference frame.

Although the findings are clear to this point, there are some aspects of these data that do not match the predictions and warrant further discussion. One concerns the pattern of data on the static square condition: although we see an increase in the severity of neglect as probability goes from $20 \%$ to $80 \%$ in both the static and moving circles and in the moving squares conditions, this is not the case in the static squares. Increasing the probability of the target in the locationbased frame does not significantly affect the neglect severity in the squares in the static condition, although the increase in RT of $34 \mathrm{~ms}$ in the $80 \%$ over the $20 \%$ sampling is in the same direction as for the other conditions. We suspect that this failure to observe the probability effects in this one condition may be one of inadequate statistical power, but this remains to be determined in future work.

Another puzzling aspect of the data concerns the RTs on the right-sided targets in the frequently probed frame of reference. If it is the case that the 'gain' is turned up on the competition, then one might have predicted that just as the contralesional high-probability targets are disadvantaged, so the RTs to the ipsilesional high-probability targets would have been facilitated. This was not obviously so although a detailed examination of the data reveal the following: the mean RT to the right circle in the static condition with $20 \%$ probing is $599 \mathrm{msec}$ whereas it is 556 msec with $80 \%$ probing, revealing a 43 ms difference. A comparison of the RTs for the squares, collapsed across static and moving conditions, reveals a median time of $586 \mathrm{msec}$ and $564 \mathrm{msec}$ for the
$20 \%$ and $80 \%$ conditions, respectively, and a difference of $22 \mathrm{msec}$. Although neither of these effects reach significance, they are both in the correct direction. The lack of significance, as in the case above, might arise from the reduced power in such a small sample of subjects. Alternatively, it is possible that subjects are operating at the limits of their ability and we are observing a floor effect. These are elderly braindamaged subjects and it may be that their RTs cannot speed up much more.

In sum, this experiment has confirmed that neglect is a deficit of attention, rather than one of perceptual representations. In fact, the findings suggest that subjects are able to attend preferentially to the right side of an object, irrespective of its spatial location, while at the same time selectively attending to the right of a location within which the object appears. Furthermore, we have demonstrated that subjects are able to orient attention flexibly to particular frames of reference, depending upon the likelihood of a target's appearance. The pathology of attention, revealed by neglect, is most salient in the frame of reference towards which attention is oriented.

\section{General Discussion}

These experiments were designed to determine whether the deficit in processing contralesional stimuli shown by patients with unilateral neglect can be observed in two different frames of reference simultaneously. More specifically, we wished to determine whether object-centered neglect might be observed concurrently with neglect in a different set of spatial coordinates. The experiments were motivated by three factors: First, consideration of perceptual processes clearly shows that 
objects are not perceived in isolation, but rather, object perception is determined by the visual background environment (e.g., J. J. Gibson, 1979). Second, attentional mechanisms function in both spatial- and object-based frames simultaneously in normal subjects in the same task (Egly et al., 1994; Egly et al., 1994) or their relative contribution can be influenced by task instructions (Vecera \& Farah, 1994). And third, in neuropsychological patients, there is suggestive evidence that neglect can be observed in two frames of reference, but that the extent to which neglect manifests may depend upon the behavioral requirements of the task (Humphreys \& Riddoch, 1994; Humphreys \& Riddoch, 1995; Riddoch et al., 1995; Riddoch et al., 1995).

\section{Multiple spatial representations}

What we have demonstrated for the first time is that patients with visual neglect, like normal subjects (Egly et al., 1994), represent information in both location- and object-centered frames simultaneously in the same task (see also Behrmann \& Moscovitch, 1994). Thus, even when targets are presented in close spatial proximity on the same side of a visual display, detection performance can be quite different. For example, after the barbell has rotated, detection of targets on the left side of space differs depending on the reference frame: if the target falls on the right side of the barbell, performance is relatively good, but if the target falls on the left defined by a set of location coordinates, detection is impaired. On the other hand, targets spaced far apart and in different hemifields can have fairly similar detection performance: detection of targets appearing in the left square and targets appearing in the barbell on the right side of space after rotation produces patterns of behavior that are not statistically different from each other. These results cannot be explained by any model of attention that argues solely for a location-based medium. The findings also cannot be explained by a view in which information on the left does not reach awareness because the contralesional spatial medium is poorly resolved and unable to support the item as an independent token (see, for discussion of this point, Farah, 1994). Rather, the current findings can only be explained by assuming that the positions of targets are defined with respect to particular frames of reference, and that spatial position (and neglect thereof) is determined with respect to these frames.

A further issue addressed in this paper concerns whether the distribution of attention in these two frames of reference is fixed and invariant, or whether it may be flexible, and influenced by task demands (Humphreys \& Riddoch, 1995; Vecera \& Farah, 1994). By manipulating the probability of targets appearing within the mobile object or the static loci, we have shown that the pattern of neglect also varied. Specifically, as the probability of a target appearing in the mobile object was increased, so the severity of neglect, reflected as a difference between detection of left and right targets, also increased. The increase in neglect with more frequent sampling in a frame was also observed in the squares but was more clearly seen when the barbell was moving than when the entire display was static. These results provide further support for the notion that neglect is a pathology of attention, because the nature of the neglect, as determined by which frame of reference was dominant, is substantially altered by subject's attentional strategies (Baylis et al., 1993).

The finding that spatial information is represented in more than 
one coordinate system is consistent with considerable data from single unit recordings in parietal cortex of nonhuman primates. For example, in relatively early work, Andersen and colleagues (Andersen, Essick, \& Siegel, 1985; Andersen \& Mountcastle, 1983) showed that single cells in parietal cortex have retinal receptive fields and, moreover, the firing of these cells is modulated by the orbital position of the eyes (see also, Colby, 1996; Duhamel, Colby, \& Goldberg, 1992). This convergence of the eye and orbital signal provides a means for representing space in a head-centered frame. Furthermore, the activity of cells in the same area of parietal cortex is modulated by the position of the head relative to the trunk (through vestibular and/or proprioceptive feedback) and this contributes to a representation that is trunk-centered or defined with respect to the body midline (Brotchie, Andersen, Snyder, \& Goodman, 1995). Finally, input from vestibular signals to these cells provide information about the orientation of the head in the world (Snyder, Brotchie, \& Andersen, 1993) and, combined with the retinal and orbital information, produces a spatial code in world- (environment or location, in our terminology) coordinates (see Andersen, Snyder, Bradley, \& Xing, 1997; Andersen, Snyder, Li, \& Stricanne, 1993; Colby, 1997, for overviews of this research). Recently, Olson and Gettner (Olson \& Gettner, 1998) have demonstrated a deficit object-centered directional selectivity in monkeys with lesions to parietal cortex (Olson \& Gettner, 1996). That information in parietal cortex is coded with respect to many different sets of coordinates predicts that, following brain-damage to this region, a spatial deficit such as neglect should be observed for information on the contralateral side in multiple frames of reference. This prediction is further supported by computational simulations which have shown that damage to parietal neurons which perform sensorimotor transformations can give rise to neglect in multiple different coordinates (Pouget \& Sejnowski, 1997).

We have concluded that spatial information is thus represented in multiple frames of reference and that damage to parietal cortex reflects these representations. An alternative explanation that must be addressed before definitively concluding that it is the multiple spatial representations that ultimately determine neglect behavior, however, has to do with whether the data we have reported are simply an artifact of eye movements. Such an explanation might work as follows: the neglect subjects initially fixate the right of the barbell because their attention is preferentially distributed to that side in the preview display and then, when the barbell starts rotating, they track the movement of the right circle. When the barbell finally stops, the subjects will now be fixating the left side of space which is occupied by the right of the barbell. The speed-up of left detection in the moving condition relative to the static condition might then emerge from the fact that subjects are now fixating the position in which the left target will appear. The observed facilitation then is simply a product of a shift in overt fixation and is unrelated to the object per se. On this same account, the slowing to detect the target on the right side comes from the fact that the subjects are fixating far over to the left of the display and would need to move their eyes and/or their covert attention to the right. This would take time and a RT cost, giving rise to the observed inhibition for 
right-sided targets in the moving condition.

This eye movement explanation, however, does not provide an adequate account of the data for two main reasons. In the first instance, Tipper and Behrmann (1996; Experiment 2) tested this alternative hypothesis directly in two neglect subjects using the same static and rotating barbell paradigm as that employed here while simultaneously monitoring their eye movements. Both of the hallmarks of object-centered neglect, the left-sided facilitation and right-sided inhibition, were observed in these two subjects even when eye movements were held constant. These findings rule out eye movements as a possible explanation. A second compelling reason for ruling out eye movements comes from the data obtained in this paper. If subjects were simply tracking the right circle of the barbell while it rotates into the left side of space, then we might have expected that detection of the target in the square on the left side, now adjacent to fixation and the focus of attention, should also have been facilitated in the moving condition. This, however, was not the case and detection of the target in the left square is both poor in the moving condition and equally so in the static condition (see Figure 4 and Figure 6 Panel B). What is observed instead (and to an equivalent extent in the moving and static condition) is that detection of the target in the right square, located 14.3 degrees away from the hypothesized fixation, was relatively good. These findings provide further evidence for the claim that eye movements are not responsible for the patterns of behavior in these experiments. Rather, the findings are compatible with the claim that spatial information may be represented in two different reference frames and that neglect may be observed in both simultaneously.

What are the exact reference frames? Although we have established that spatial information may be represented in multiple frames of reference, we need to examine exactly what those reference frames are. We will consider the squares and circles in turn. We have couched the argument in terms of a contrast between location-based and object-based attention but one might argue that the distinction is better cast as between two objects, one foreground and one background. The barbell would constitute the foreground object as we have already proposed, but now the squares would form part of a single background object and not constitute a location-based representation. Under this interpretation, there is only neglect for the left of object-based representations with two independent representations for the foreground and background. Given the limitations that normal subjects display when processing more than one object at a time (Duncan, 1984; Neisser, 1967), we think this hypothesis is unlikely. Also, it is our intuition that if we presented a display consisting of only two squares to normal subjects, they would be unlikely to consider them as a single object. We favor the interpretation, therefore, that the squares are part of a non-object based representation although, as discussed previously, there are several potential contenders for what that representation might really be. An important implication of our perspective, however, is that, while subjects may be impaired at switching between two objects (Duncan, 1984), there is minimal or no cost in switching between two reference frames. This hypothesis awaits further empirical verification. 
If we consider the barbell now, we have argued that the circles are coded in an object-based representation. Exactly what this means is open to different interpretations. At the most basic level, one might argue that the barbell is not an object per se but is rather a grouped set of contours that move together and share Gestalt properties such as good continuation and common fate (Driver \& Baylis, 1989). We would not argue with this interpretation and certainly acknowledge that the two circles and horizontal bar of the barbell are grouped together. In our view, this is exactly what constitutes an object-based representation and, over time, with increased experience and familiarity with the stimulus, and as it is imbued with meaning, so it comes to have a long-term object representation (for such a scheme, see Mozer, Zemel, Behrmann, \& Williams, 1992). For the current purposes, however, the two views, of a grouped display, and an object, are equivalent.

But is the 'object' then simply defined by its initial egocentric encounter? Driver and colleagues have argued that the relative left and right of an object are defined relative to a midline axis imposed from the egocentric perspective and thus there is nothing special or 'object'-like about the stimulus (Driver et al., 1994). This is in contrast with objects which have a canonical or intrinsic handedness such as maps or words and which have a 3D or object-representation, defined in a Marrian sense (Marr \& Nishihara, 1978). In previous work, one of us has shown that patients with neglect omit to report letters that appear on the left of objects (letters of the alphabet) that have an intrinsic asymmetry, irrespective of the orientation of the object (Behrmann $\&$ Moscovitch, 1994). In the context of the previous study, whether the object- based neglect arises from a canonical 3D representation of the barbell object or from a representation defined by the viewer which then moves along with the object, remains an open issue. The barbell is colored asymmetrically and subjects who see a blue circle on the left will always see the blue circle on that side. During the course of the experiment, then, the barbell may come to have a canonical representation with the blue circle on its intrinsic left, irrespective of the orientation of the barbell. Thus, even though the left of an object might be assigned during a particular initial encounter with it, this is still an object qualitatively different from the background space in which it exists. Moreover, over the course of processing, a 3D representation might be formed and this might mediate subsequent performance.

\section{Attention as competition}

A particularly counterintuitive aspect of the findings obtained in these experiments is that the severity of neglect is increased when the probability of a particular frame being probed is increased. This finding is difficult to interpret on some standard accounts of attention which have proposed that the main function of attention is to facilitate perceptual processing (Broadbent, 1958) and that attending to a stimulus should therefore yield faster RTs. We have suggested, however, that the increase in neglect is compatible with views of attention in which items compete for selection. When one item is disadvantaged, as in the case of the left items in patients with right hemisphere lesions, this item will lose out more often. The time to detect and process it, then, will be substantially increased.

There are two implemented mechanistic accounts which incorporate such a competitive mechanism in the 
context of spatial attention and both exhibit performance consistent with the view proposed here. Cohen, Romero, Servan-Schreiber, \& Farah, (1994), for example, have explicitly demonstrated in the context of a neural network model that attention emerges from competition between left and right stimuli and that the failure to process the left-sided stimulus revealed by neglect patients may be captured by imposing a negative spatial bias onto the units representing the left side. Similarly, Mozer and colleagues (Mozer \& Behrmann, 1990; Mozer, Halligan, \& Marshall, 1997) have 'lesioned' a neural network, previously designed to simulate aspects of normal attentional behavior, by imposing a gradient of probability across the connections from the retinal or input layer to the attentional mechanism (AM). The probability with which the left-sided information is selected is a function of this gradient; if the gradient is less steep such that left-sided information is being activated to some extent, neglect would less severe. The more steep the gradient, the more likely the right-sided information will be selected as 'winner' and the more severe the neglect.

A possible neural basis for the exaggeration of neglect as attention to a particular frame of reference is increased is one of greater neural activity associated with behavioral intention (Bushnell, Goldberg, \& Robinson, 1981; Colby, 1996). These increasingly active neurons set the threshold for the competition and increase the differential between information on the left and right, resulting in greater suppression of contralateral stimuli. In the context of the experiments reported here, then, an increase in the extent of left-sided neglect arises from increased competition in the frequently probed reference frame and this leads to a heightened asymmetry in the more dominant frame.

Support from 'extinction' for the competition hypothesis of neglect

We have claimed that a view of selective attention as the outcome of a competitive mechanism can account for the findings obtained in this paper. This view has also received increasing support from a host of other recent studies with brain-damaged patients with lesions in parietal cortex who show 'extinction'. 'Extinction' refers to the pattern of performance in which patients are significantly impaired at processing information on the contralesional left side, but only when this information is presented simultaneously with ipsilesional right-sided information. For example, patients AC and GB (Ward, Goodrich, \& Driver, 1994), reported the presence of a single bracket or single dot on the left side correctly on approximately $75 \%$ and $95 \%$ of the trials, respectively. When this left side information was presented concurrently with right-sided information, report of the single dot or bracket fell to about $25 \%$ and $0 \%$ for the two patients. Interestingly, the competition between two stimuli, one on the left and one on the right, and extinction of the left stimulus can even be observed when the two stimuli are presented sequentially but within $600 \mathrm{msec}$ of each other (di Pellegrino, Basso, \& Frassinetti, 1997).

The impairment in contralesional processing only when competing ipsilesional information is present contrasts with the pattern in neglect in which information appearing even alone on the contralesional side is poorly processed. There are other, important differences between the extinction and neglect patients. In extinction, as several recent studies have demonstrated, the impaired processing of the 
contralesional information in the doubleitem displays can be offset if this contralesional information can be bound or grouped together with the ipsilesional information. For example, Ward et al. (1994) showed that when the contralesional item could be grouped with the ipsilesional information on the basis of Gestalt factors such as similarity (for example, a bracket on the left and a bracket on the right) or symmetry, report of the left-sided stimulus improved by roughly $50 \%$ for both $\mathrm{AC}$ and $\mathrm{GB}$ compared to when the left sided information could not be grouped with a simultaneous right sided stimulus. The same pattern was obtained when the two items formed a familiar configuration (for example, an arrow made of a left arrowhead and a right horizontal bar). This modulation of extinction of the contralesional information has suggested that, in the context of a competitive mechanism, the negative bias for the contralesional information is reduced such that contralesional and ipsilesional information form a single group and cooperate rather than compete.

The reduction of extinction through grouping has now been replicated in several studies with parietal patients and better processing of the contralesional information has been shown when the left-sided information can be grouped by bottom-up factors such as color and proximity (Driver \& Halligan, 1991), brightness or collinear edges (Gilchrist, Humphreys, \& Riddoch, 1996; Rorden, Mattingley, Karnath, \& Driver, 1997). A reduction in contralesional extinction is also seen when the left information is grouped with the right information by a global outline (Driver, Baylis, \& Rafal, 1992; Farah, Wallace, \& Vecera, 1993), by an illusory contour (Kanizsa-type figure) of a partially occluded figure (Mattingley, David, \& Driver, 1997), or by any well- configured object or whole (Gilchrist et al., 1996; Humphreys \& Riddoch, 1994). Top down effects also play a role with less extinction for known, familiar objects or words than for unknown objects or words (Behrmann, Moscovitch, Black, \& Mozer, 1990); Driver, Baylis, \& Rafal, 1992; Ward \& Goodrich, 1996). Similar effects of grouping have been observed with normal subjects in which unattended information is processed better if it forms part of an object defined by uniform connectedness (Kramer \& Hahn, 1996; Watson \& Kramer, 1998).

Given that connecting or grouping of a left- and right-sided item into a single object can partially offset the poor contralesional processing in parietal patients, it is curious that we continue to see significantly poorer detection of the left-sided target in the present study in which the left and right circles group to form a single barbell. If left-sided information is grouped with the right, then we should not observe such poor contralesional processing in the object-centered frame. In fact, one might even expect to see especially strong grouping effects in our study in which the right and left sides of the barbell move together given that motion and common fate have been shown to be particular robust in producing perceptual organization effects (Driver \& Baylis, 1989). Furthermore, in a previous study in which we specifically examined the severity of neglect when the circles were joined by a horizontal bar to form a barbell and when they were not connected, we saw the object-centered effect only in the grouped or joined and not in the disconnected barbell condition (Tipper \& Behrmann, 1996). This finding would appear to contradict those data that suggest that grouping the left stimulus with the right improves its detection. 
There does not seem to be an obvious solution to these contradictory findings but a number of possible explanations can be proposed. One explanation concerns a possible difference between the patients who have participated in the different studies. The patients who participated in the studies reported here as well as in our other studies have all shown explicit signs of neglect and omit left-sided information on pencil and paper tests (see Figure 3). Some, although not all of the patients in the extinction studies perform relatively well on these standard tests of neglect. For example, patient GB (Ward \& Goodrich, 1996; Ward et al., 1994), patient VR (Mattingley et al., 1997, footnote 17; Rorden et al., 1997) and patient EN (Rorden et al., 1997) exhibit mild (if any) neglect on these standard neglect measures. The differences between these patients might lead one to suggest that extinction and neglect are qualitatively different phenomena and hence principles of grouping and connectedness might operate in the case of the former but not the latter. An alternative is that these are simply quantitative variations of the same basic deficit. Indeed, extinction has often been considered to be a milder form of neglect or a stage in the recovery from the more severe neglect deficit (Critchley, 1953; McFie \& Zangwill, 1960). If the difference between patients is simply a matter of degree of deficit, it is not surprising that extinction patients but not neglect patients are positively influenced by grouping. In the case of the extinction patients, left-sided information is activated to some extent; although the left-sided information is not selected during the course of the competition between potential targets when there is both a left and right target present simultaneously, subjects can still take advantage of its activation and exploit it in the context of grouping. In contrast, in the case of the neglect patients, the contralesional stimulus is so poorly activated that even when it is presented alone, subjects fail to detect its presence. In such a case, because of the minimal activation, subjects cannot use left sided information to take advantage of perceptual aspects of the display such as grouping or of top-down information such as lexical knowledge (for a similar severity argument and its computational implementation, see Behrmann et al., 1990, and Mozer \& Behrmann, 1990).

This view argues that extinction and neglect might simply be parametric variations of the same basic phenomenon and that the former but not the latter can exploit perceptual and conceptual aspects of the stimulus display. This severity argument might still apply to those few extinction patients who show effects of grouping but who also perform poorly on neglect tests. It might still be the case that these patients (for example, GK, Gilchrist et al., 1996; Humphreys et al., 1994; Humphreys, personal communication; and patient AC of Ward et al., 1994) are more mildly affected than those patients with florid neglect who have participated in our studies and have some residual activation that can be exploited under conditions of grouping.

A second possible explanation for the improved reporting of left-sided information in some studies but not in the studies using the barbell paradigm may have to do with methodological differences between the paradigms. For example, Ward et al. (1994) and Mattingley et al. (1997) presented stimuli for a limited exposure duration; in the former case, stimuli were presented for a duration that ranged between $43 \mathrm{~ms}$ and $129 \mathrm{~ms}$ (with masking) and detection accuracy was 
measured. In contrast, in our studies, the barbell and target remained on the screen for an extended time of $3 \mathrm{~s}$, very few errors were made and RT to detect the target probe was measured. A second methodological difference concerns the tasks the subjects performed. In those studies which show the grouping advantage, the subjects directly report the presence of the stimulus; for example, subjects report whether they observed the presence of the left-sided bracket or arrowhead (Ward et al., 1994) or the pac-man shapes in the illusory contour experiment (Mattingley et al., 1997). In our study, the barbell only provides the background context upon which the target is superimposed and subjects do not directly report the presence of the circle. One obvious test of this difference in paradigms would be to have subjects in the barbell paradigm explicitly report the presence of the circle both in the static and moving condition in a situation in which the circle is present on only some subset of the displays. If the difference between the studies is simply a function of task demands, we would predict that under these circumstances, we would obtain the grouping effect such that the left of the barbell would be better detected when it was connected with the right of the barbell compared to when the two circles were disconnected. That differences in methodological procedure can produce different patterns of results for object- and location-based forms of attention has already been suggested by (Lavie \& Driver, 1996).

Conclusion
The brain is capable of
representing visual information in

multiple frames of reference, ranging from retinotopic, environment-based, object-centered, and even actioncentered (Tipper, Lortie, \& Baylis, 1992). It follows therefore, that attentional systems required to select perceptual representations for selective action should also be able to access a variety of reference frames depending upon behavioral goals. Furthermore, when those behavioral goals require a response to information emerging from two frames of reference in an unpredictable manner, then attention must gain access to both frames.

Evidence from single unit recordings with nonhuman primates have demonstrated that these different representations are all mediated by neurons in parietal cortex (Andersen et al., 1997; Colby, 1998; Olson \& Gettner, 1998). The findings reported here have confirmed that, following a lesion to parietal cortex, information appearing on the relative left defined with respect to multiple reference frames is less well attended and selected than information on the right. Specifically, unilateral neglect, assumed to be a pathology of attention, has been revealed in both location-based and object-centered frames of reference simultaneously. Furthermore, as predicted by contemporary models which emphasize competition as the neural mechanism which instantiates selection, attention can be biased in favor of particular reference frames via task contingencies. In the context of neglect, greater activation of a reference frame results in greater levels of reactive inhibition, producing more severe patterns of neglect. 


\section{References:}

Abrams, R. A., \& Dobkin, R. S. (1994). Inhibition of return: Effects of attentional cuing on eye movement latencies. Journal of Experimental Psychology: Human Perception and Performance, 20, 467-477.

Albert, M. L. (1973). A simple test of visual neglect. Neurology, 23, 658-664.

Andersen, R. A., Essick, G. K., \& Siegel, R. M. (1985). Encoding of spatial location by posterior parietal neurons. Science, 230, 456-458.

Andersen, R. A., \& Mountcastle, V. B. (1983). The influence of the angle of gaze upon the excitability of the light-sensitive neurons of the posterior parietal cortex. Journal of Neuroscience, 3, 532-548.

Andersen, R. A., Snyder, L. H., Bradley, D. C., \& Xing, J. (1997). Multimodal representation of space in the posterior parietal cortex and its use in planning movements. Annual Review Neuroscience, 20, 303-330.

Baylis, G., Driver, J., \& Rafal, R. D. (1993). Visual extinction and stimulus repetition. Journal of Cognitive Neuroscience, 5(4), 453-466.

Baylis, G. C., \& Driver, J. (1992). Visual parsing and response competition: The effect of grouping factors. Perception and Psychophysics, 51(2), 145-162.

Baylis, G. C., \& Driver, J. (1993). Visual attention and objects: Evidence for hierarchical coding of location. Journal of Experimental Psychology: Human Perception and Performance, 19, 451-470 .

Behrmann, M., \& Moscovitch, M. (1994). Object-centered neglect in patients with unilateral neglect: Effects of left-right coordinates of objects. Journal of Cognitive Neuroscience, 6(1), 1-16.

Behrmann, M., Moscovitch, M., Black, S. E., \& Mozer, M. (1990). Perceptual and conceptual factors in neglect dyslexia: Two contrasting case studies. Brain, 113(4), 11631883.

Behrmann, M., \& Tipper, S. P. (1994). Object-based attentional mechanisms: Evidence from patients with unilateral neglect. In C. Umilta \& M. Moscovitch (Eds.), Attention and Performance XV: Conscious and nonconscious information processing (pp. 351375). Cambridge, MA: MIT Press.

Behrmann, M., Zemel, R. S., \& Mozer, M. C. (1998). Object-based attention and occlusion: Evidence from normal subjects and a computational model. Journal of Experimental Psychology: Human Perception and Performance, in press. 
Bisiach, E., Capitani, E., \& Porta, E. (1985). Two basic properties of space representation in the brain. Journal of Neurology, Neurosurgery and Psychiatry, 48, 141144.

Bisiach, E., \& Vallar, G. (1988). Hemineglect in humans. In F. Boller \& J. Grafman (Eds.), Handbook of Neuropsychology (Vol. 1, pp. 195-222). Amsterdam: Elsevier Science Publishers, Biomedical Division (North-Holland).

Black, S. E., Ebert, P., Leibovitch, F., Szalai, J., Blair, N., \& Bondar, J. (1994). Recovery in hemispatial neglect. Neurology, 45, A178.

Black, S. E., Vu, B., Martin, D., \& Szalai, J. P. (1990). Evaluation of a bedside battery for hemispatial neglect in acute stroke. Journal of Clinical and Experimental Neuropsychology, 12, 102 (abstract).

Broadbent, D. (1982). Task combination and selective intake information. Acta Psychologica, 50, 253-290.

Broadbent, D. E. (1958). Perception and Communication. London: Pergamon Press.

Brotchie, P. R., Andersen, R. A., Snyder, L. H., \& Goodman, S. J. (1995). Head position signals used by parietal neurons to encode locations of visual stimuli. Nature, 375, 232235.

Bushnell, M. C., Goldberg, M. E., \& Robinson, D. L. (1981). Behavioral enhancement of visual responses in monkey cerebral cortex: I. Modulation in posterior parietal cortex related to selective visual attention. Journal of Neurophysiology, 46, 755-771.

Buxbaum, L. J., Coslett, H. B., Montgomery, M. W., \& Farah, M. J. (1996). Mental rotation may underlie apparent object-based neglect. Neuropsychologia, 34(2), 113-126.

Caramazza, A., \& Hillis, A. E. (1990). Spatial representation of words in the brain implied by studies of a unilateral neglect patient. Nature, 346, 267-269.

Caramazza, A., \& Hillis, A. E. (1990a). Levels of representation, co-ordinate frames and unilateral neglect. Cognitive Neuropsychology, 13(5/6), 391-446.

Cohen, J., Romero, R., Servan-Schreiber, D., \& Farah, M. J. (1994). Mechanisms of spatial attention: The relation of macrostructure to microstructure in parietal neglect. Journal of Cognitive Neuroscience, 6(4), 377-387.

Colby, C. (1996). A neurophysiological distinction between attention and intention. In T. Inui \& J. L. McClelland (Eds.), Attention and Performance VXI (pp. 157-177). Cambridge, MA: MIT Press.

Colby, C. (1997). Multiple spatial reference frames in parietal cortex. Neuron, in press. 
Costello, A. D., \& Warrington, E. K. (1987). The dissociation of visual neglect and neglect dyslexia. Journal of Neurology, Neurosurgery and Psychiatry, 50, 1110-1116.

Critchley, M. (1953). The Parietal Lobes. London: Hafner Press.

Cubelli, R., Nichelli, P., Bonito, V., Tanti, A. D., \& Inzhagi, M. (1991). Different patterns of dissociation in unilateral spatial neglect. Brain and Cognition, 15, 139-159.

D'Erme, P., Robertson, I., Bartolomeo, P., Daniele, A., \& Gainotti, G. (1992). Early rightwards orienting of attention on simple reaction time performance in patients with left-sided neglect. Neuropsychologia, 30(11), 989-1000.

Desimone, R., \& Duncan, J. (1995). Neural mechanisms of selective visual attention. Annual Review of Neuroscience, 18, 193-197.

di Pellegrino, G., Basso, G., \& Frassinetti, F. (1997). Spatial extinction on double asynchronous stimulation. Neuropsychologia, 35(9), 1215-1223.

Driver, J., \& Baylis, G. C. (1989). Movement and visual attention: The spotlight metaphor breaks down. Journal of Experimental Psychology: Human Perception and Performance, 15, 448-456.

Driver, J., Baylis, G. C., Goodrich, S., \& Rafal, R. D. (1994). Axis-based neglect of visual shape. Neuropsychologia, 32(11), 1353-1365.

Driver, J., \& Halligan, P. W. (1991). Can visual neglect operate in object-centered coordinates: An affirmative study. Cognitive Neuropsychology, 8, 475-496.

Duhamel, J. R., Colby, C. L., \& Goldberg, M. E. (1992). The updating of representations of visual space in parietal cortex by intended eye movements. Science, 225, 90-92.

Duncan, J. (1984). Selective attention and the organization of visual information. Journal of Experimental Psychology: General, 113(4), 501-517.

Duncan, J. (1996). Cooperating brain systems in selective perception and action. In T. Inui \& J. L. McClelland (Eds.), Attention and Performance VXI (pp. 549-578). Cambridge, MA: MIT Press.

Duncan, J., \& Humphreys, G. W. (1989). Visual search and stimulus similarity. Psychological Review, 96(3), 433-458.

Duncker, K. (1929). Induced motion. In W. D. Ellis (Ed.), A source book of gestalt psychology (pp. 161-172). New York: Humanities Press.

Egeth, H., \& Yantis, S. (1997). Visual attention: Control, representation and time course. Annual review of Psychology, 48, 269-297. 
Egly, R., Driver, J., \& Rafal, R. (1994). Shifting visual attention between objects and locations: Evidence from normal and parietal lesion subjects. Journal of Experimental Psychology: General, 123, 161-177.

Egly, R., Rafal, R., Driver, J., \& Starrveld, Y. (1994). Covert attention in the split brain reveals specialization for object-based attention. Psychological Science, 5(6), 380-383.

Eriksen, B. A., \& Eriksen, C. W. (1974). Effects of noise letters upon the identification of a target letter in a nonsearch task. Perception and Psychophysics, 16(1), 143-149.

Eriksen, C. W., \& Hoffman, J. (1972). Temporal and spatial characteristics of selective encoding from visual displays. Perception and Psychophysics, 12, 201-204.

Eriksen, C. W., \& Schultz, D. W. (1979). Information processing in visual search: A continuous flow conception and experimental results. Perception and Psychophysics, 25, 249-263.

Farah, M. J., Brunn, J. L., Wong, A. B., Wallace, M., \& Carpenter, P. (1990). Frames of reference for the allocation of spatial attention: Evidence from the neglect syndrome. Neuropsychologia, 28, 335-347.

Farah, M. J., Wallace, M., \& Vecera, S. P. (1993). "What" and "where" in visual attention: Evidence from the neglect syndrome. In I. H. Robertson \& J. C. Marshall (Eds.), Unilateral neglect: Clinical and Experimental Studies (pp. 123-138). Hove, UK: Lawrence Erlbaum Associates.

Gauthier, L., Dehaut, F., \& Joanette, Y. (1989). The bells test: A quantitative and qualitative test for visual neglect. International Journal of Clinical Neuropsychology, $X I(2), 49-54$.

Gibson, B. S., \& Egeth, H. (1994). Inhibition of return to object-based and environmentbased locations. Perception and Psychophysics, 55(3), 323-339.

Gibson, J. J. (1979). The Ecological Approach to Visual Perception. Boston: HoughtonMifflin.

Gilchrist, I. D., Humphreys, G. W., \& Riddoch, M. J. (1996). Grouping and extinction: Evidence for low-level modulation of visual selection. Cognitive Neuropsychology, 13(8), 1223-1249.

Goldstein, E. B. (1996). Sensation and perception. (Fourth ed.). Pacific Grove, CA: Brooks/Cole.

Hillis, A. E., \& Caramazza, A. (1995). A framework for interpreting distinctive patterns of hemispatial neglect. Neurocase, 1, 189-207. 
Hoffman, J. E., \& Nelson, B. (1981). Spatial selectivity in visual search. Perception and Psychophysics, 30, 283-290.

Humphreys, G. W., \& Riddoch, M. J. (1994). Attention to within-object and betweenobject spatial representations: Multiple sites for visual selection. Cognitive Neuropsychology, 11(2), 207-241.

Humphreys, G. W., \& Riddoch, M. J. (1995). Separate coding of space within- and between perceptual objects: Evidence from unilateral visual neglect. Cognitive Neuropsychology, 12(3), 283-312.

Humphreys, G. W., Romani, C., Olson, A., Riddoch, M. J., \& Duncan, J. (1994). Nonspatial extinction following lesions of the parietal lobe in humans. Nature, 372, 357-359.

Kahneman, D., \& Treisman, A. (1984). Changing views of attention and automaticity. In R. Parasuraman \& D. R. Davies (Eds.), Varieties of attention (pp. 29-61). New York: Academic Press.

Karnath, H. O., Schenkel, P., \& Fisher, B. (1991). Trunk orientation as the determining factor of the contralateral deficit in the neglect syndrome and as the physical anchor of the internal representation of body orientation in space. Brain, 114, 1997-2014.

Kramer, A. F., \& Hahn, S. (1996). Splitting the beam: Distribution of attention over noncontiguous regions of the visual field. Psychological Science, 6(6), 381-386.

Kramer, A. F., \& Jacobson, A. (1991). Perceptual organization and focused attention: The role of objects and proximity in visual processing. Perception and Psychophysics, 50, 267-284.

Kramer, A. F., \& Watson, S. E. (1996). Object-based visual selection and the principles of uniform connectedness. In A. F. Kramer \& G. Logan (Eds.), Converging operations in the study of visual selective attention (pp. 395-414). Washington, DC: APA.

Kramer, A. F., Weber, T. A., \& Watson, S. E. (1997). Object-based attentional selection - Grouped-arrays or spatially-invariant representations? Comment on Vecera and Farah (1994). Journal of Experimental Psychology: General, 126, 3-13.

Làdavas, E. (1987). Is hemispatial deficit produced by right parietal damage associated with retinal or gravitational coordinates. Brain, 110, 167-180.

Làdavas, E., Petronio, A., \& Umilta, C. (1990). The deployment of visual attention in the intact field of hemineglect patients. Cortex, 26, 307-317.

Lavie, N., \& Driver, J. (1996). On the spatial extent of attention in object-based selection. Perception and Psychophysics, 58(8), 1238-1251. 
Marr, D., \& Nishihara, H. K. (1978). Representation and recognition of the spatial organization of three-dimensional shapes. Proceedings of the Royal Society of London, Series B, 200, 269-294.

Matin, L., Picoult, E., Stevens, J., Edwards, M., \& MacArthur, R. (1982). Oculoparalytic illusion: Visual-field dependent spatial mislocalizations by humans partially paralysed by curare. Science, 216, 198-201.

Mattingley, J. B., David, G., \& Driver, J. (1997). Pre-attentive filling in of visual surfaces in parietal extinction. Science, 275, 671-674.

McFie, J., \& Zangwill, O. L. (1960). Visuo-constructive disabilities associated with lesions of the left cerebral hemisphere. Brain, 82, 243-260.

Moore, C., Yantis, S., \& Vaughan, B. (1998). Object-based visual selection: Evidence from perceptual completion. Psychological Science, 9(2), 104-110.

Mozer, M. C., \& Behrmann, M. (1990). On the interaction of selective attention and lexical knowledge: A connectionist account of neglect dyslexia. Journal of Cognitive Neuroscience, 2(2), 96-123.

Mozer, M. C., Halligan, P. W., \& Marshall, J. C. (1997). The end of the line for a braindamaged model of hemispatial neglect. Journal of Cognitive Neuroscience, 9(2), 171190.

Mozer, M. C., Zemel, R., Behrmann, M., \& Williams, C. (1992). Learning to segment by dynamic feature binding. Neural Computation, 4, 647-672.

Neisser, U. (1967). Cognitive Psychology. New York: Appleton Century Crofts.

Olson, C., \& Gettner, S. (1998, ). Impairment of object-centered vision following lesions of macaque posterior parietal cortex. Paper presented at the Soc. for Neuroscience Abstr.

Olson, C., \& Gettner, S. N. (1996). Brain representation of object-centered space. Current Opinion in Neurobiology, 6, 165-170.

Posner, M. I. (1980). Orienting of attention. Quarterly Journal of Experimental Psychology, 32, 3-25.

Posner, M. I., \& Cohen, Y. (1984). Components of performance. In H. Bouma \& D. Bouwhuis (Eds.), Attention and Performance $X$ (pp. 531-556). Hillsdale, NJ: Lawrence Erlbaum Associates.

Pouget, A., \& Sejnowski, T. J. (1997). Spatial transformations in the parietal cortex using basis functions. Journal of Cognitive Neuroscience, 9(2), 222-237. 
Reuter-Lorenz, P., Drain, M., \& Hardy-Morais, C. (1996). Object-centered attentional biases in the normal brain. Journal of Cognitive Neuroscience, 8(6), 540-550.

Riddoch, M. J., Humphreys, G., Burroughs, E., Luckhurst, L., Bateman, A., \& Hill, S. (1995). Cueing in a case of neglect: Modality and automaticity effects. Cognitive Neuropsychology, 12(6), 605-622.

Riddoch, M. J., Humphreys, G. W., Luckhurst, L., Burroughs, E., \& Bateman, A. (1995). "Paradoxical neglect": Spatial representations, hemisphere-specific activation and spatial cueing. Cognitive Neuropsychology, 12(6), 569-604.

Rorden, C., Mattingley, J. B., Karnath, H.-O., \& Driver, J. (1997). Visual extinction and prior entry: Impaired perception of temporal order with intact motion perception after unilateral parietal damage. Neuropsychologia, 35(4), 421-433.

Snyder, L. H., Brotchie, P., \& Andersen, R. A. (1993). World-centered coding of location in posterior parietal cortex of monkey. Society for Neuroscience (Abstracts), 19, 315.311.

Stuart, G. W., Maruff, P., \& Currie, J. (1997). Object-based visual attention in luminance increment detection. Neuropsychologia, 35(6), 843-853.

Tipper, S., Weaver, B., \& Houghton, G. (1994). Behavioral goals determine inhibitory mechanisms of selective attention. Quarterly Journal of Experimental Psychology, 47A, 809-840.

Tipper, S. P., \& Behrmann, M. (1996). Object-centred not scene-based visual neglect. Journal of Experimental Psychology: Human Perception and Performance, 22(5), 12611278.

Tipper, S. P., Lortie, C., \& Baylis, G. C. (1992). Selective reaching : Evidence for action-centered attention. Journal of Experimental Psychology: Human Perception and Performance, 18(4), 891-905.

Tipper, S. P., \& Weaver, B. (1996). The medium of attention: Location-based, objectcentered or scene-based? In R. Wright (Ed.), Visual attention . Oxford, UK : Oxford University Press.

Tipper, S. P., Weaver, B., Jerreat, L. M., \& Burak, A. L. (1994). Object- and environment-based inibition of return of visual attention. Journal of Experimental Psychology: Human Perception and Performance, 20, 478-499.

Tipper, S. T., Driver, J., \& Weaver, B. (1991). Object-centred inhibition of return of visual attention. Quarterly Journal of Experimental Psychology, 43A, 289-298.

Treisman, A. (1992). Perceiving and re-perceiving objects. American Psychologist, 47, 862-875. 
Tsal, Y., \& Lavie, N. (1988). Attending to color and shape: The special role of location in selective visual processing. Perception and Psychophysics, 44, 15-211.

Umilta, C., Castiello, U., Fontana, M., \& Vestri, A. (1995). Object-centred orienting of attention. Visual Cognition, 2, 165-182.

Vecera, S., \& Farah, M. J. (1994). Does visual attention select objects or locations? Journal of Experimental Psychology: Human Perception and Performance, 123(2), 1-14.

Wallach, H. (1959). The perception of motion. Scientific American, 201, 56-60.

Ward, R., \& Goodrich, S. (1996). Differences between objects and nonobjects in visual extinction. Psychological Science, 7(3), 177-180.

Ward, R., Goodrich, S., \& Driver, J. (1994). Grouping reduces visual extinction: Neuropsychological evidence for weight-linkage in visual selection. Visual Cognition, 1(1), 101-129.

Watson, S. E., \& Kramer, A. F. (1998). Object-based visual selective attention and perceptual organization. Perception and Psychophysics, in press.

Weber, T. A., Kramer, A. F., \& Miller, G. A. (1997). Selective processing of superimposed objects: an electrophysiological analysis of object-based attentional selection. Biological Psychology, 45, 159-182. 